\title{
Emergent and divergent resilience behavior in catastrophic shift systems
}

\author{
Venkatraman Srinivasan, Praveen Kumar* \\ Department of Civil and Environmental Engineering, University of Illinois at Urbana \\ Champaign, Urbana, IL 61801, USA
}

\begin{abstract}
Resilience in dynamic ecological systems has been intuitively associated with the ability to withstand disturbances in systems drivers represented as shocks. Typically shocks are characterized as an instantaneous and isolated, non-interacting events with the system dynamics corresponding to a fixed potential well. However, ecological systems are subject to continuous variation in environmental drivers such as rainfall, temperature, etc. and these interact with the ecosystem dynamics to alter the potential well. These variations are typically represented as a stochastic process. Furthermore, with climate change, the stochastic characteristics of the environmental drivers also change, thereby impacting the well dynamics. To characterize the resilience behavior under continuous variation in system drivers, and contrast it with that subject to instantaneous shock in system drivers, we employ the canonical catastrophic shift system as an example, and demonstrate emergent and contrasting divergent resilience behavior of the measures as the properties of the system-driver couple change. These behaviors include: variability induced stabilization or enhancement of dynamic regimes, regions of sensitivity to dynamic regime transitions and existence of trap or escape regions. Furthermore, we introduce the concept of iso-resilience curves which are employed to design travel paths in resilience landscapes. These re-
\end{abstract}

\footnotetext{
${ }^{*}$ Corresponding author, Address: 2527B Hydrosystems Laboratory, 301 N. Mathews Avenue, Urbana, IL-60801, Phone: (217)333-4688, Fax: (217)333-0687

Email addresses: vsriniv3@illinois.edu (Venkatraman Srinivasan), kumar1@illinois.edu (Praveen Kumar)
}

Preprint submitted to Ecological Modeling

October 14, 2013

(C) 2013. This manuscript version is made available under the Elsevier user license http://www.elsevier.com/open-access/userlicense/1.0/ 
sults provide valuable insights for managing resilience attributes associated with dynamic regime transitions in catastrophic shift systems under instantaneous shock and continuous variability in system drivers.

Keywords: vulnerability, recovery, regime change, ecosystem transitions, iso-resilience

\section{Introduction}

Catastrophic shift systems have been used as a model to explain a range of phenomenon from lake eutrophication [7, 10], shrub grass transition [7, 44], algal overgrowth in corals [5, 8], insect outbreak dynamics [50] and others [16]. The ubiquitous characteristics that these phenomena share are bi-stability, abrupt switching between alternate dynamic regimes, and hysteresis [7, 44, 16, 24, 43, 55]. Bi-stability implies that the long-term dynamics converges to one of two stable points depending on the initial condition. The set of all initial states that converge to a specific stable point is referred to as its basin or domain of attraction (DOA). When the system is stochastic in nature, then the deterministic concept of DOA is replaced by the probabilistic concept of dynamic regime $[43,6]$. Each DOA or dynamic regime is typically associated with its unique characteristic dynamics, processes, and feedbacks, such as turbid versus clear lake, shrub versus grass dominated landscape, etc. [44]. These systems have the capacity to switch to the alternate DOA or dynamic regime through a sudden or rapid transition, when system parameters, typically under the influence of external stress such as climate change, cross a critical threshold. More importantly, they exhibit a hysteresis effect, where the new dynamic regimes are sustained even after the removal of the stressor that caused the transition.

Resilience is broadly understood as the ability to withstand a change such that the dynamical behavior remains relatively unaffected [16, 24, 23, 53]. The concept of resilience has also been associated with other related and often overlapping concepts such as vulnerability, adaptability, persistence, robustness, resistance, redundancy, stability, recovery, ability to self organize, transformabil- 
ity, flexibility, and ability to learn $[7,8,16,6]$. Resilience also holds one of the key wedges in sustainability science $[7,8,6,38]$ and is increasingly being used for developing strategies to mitigate climate change [8, 16, 27, 54]. Climate scientists have predicted changes in both the mean and the extremes in future climate forcings due to anthropogenic climate change [14, 47]. Increases in climate variability, and occurrence of extreme events such as temperature extremes in the form of heat and cold waves [42, 49], increased occurrences of droughts and floods [36, 52], increased frequency of hails [32] etc. can have significant impact on ecosystem processes such as vegetation growth and mortality [22], occurrence of fires [22], patterns of herbivory [20] etc. Hence, climate change can alter ecosystem behavior by: i) directly changing the system parameters, and; ii) altering other aspects of system driver such as frequency, intensity, variability and asymmetric bias etc. [31].

An understanding of concept of resilience helps us to answer questions such as: how does a complex system absorb and respond to unexpected shock or variations in drivers [9]; what aspects of the complex systems are prone to behavioral changes under such a shock or variability $[16,6]$; what type and strength of shock or variability in the driver does it take to cause behavioral changes in particular aspects of a complex system [7]; which direction should our efforts be invested to prevent dynamic regime transitions $[8,25]$, etc. In this context, the space and time scales at which we analyze the problem play a significant role in the formulation of resilience measures [7]. We define resilience as the ability of a system's DOA or dynamic regime to maintain its structure, process and feedbacks when subject to shock or variation in drivers at a particular spatial and time scale of interest. Resilience can thus be interpreted as a higher order description of system dynamics $[6,2,15]$ that can capture characteristics of emergent behavior such as catastrophic shifts, hysteresis, dynamic regime change, etc. for which simple space and time derivative based equilibrium and stability analysis are not suitable [23].

Two of the most commonly used resilience attributes are 'engineering resilience' and 'ecological resilience' $[24,18]$. While engineering resilience is defined 
as the rate at which the system recovers to the stable state following an isolated shock in the system driver [23, 24, 16], ecological resilience is defined as the amount of shock that a system can withstand without a change in the dynamic regime $[18,51]$. More recently, there have been efforts to include other resilience attributes such as 'latitude', 'precariousness' and 'resistance' $[16,55,6]$. While the definition of latitude is similar to size of DOA, precariousness represents ecological resilience, and resistance is associated with activation potential. Several other resilience measures based on thermodynamic and information theory $[12,13]$ have also been proposed. In general, there can be multiple representations of resilience, each of which capture in general overlapping but different attributes. A particular attribute of a DOA or dynamic regime might be highly resilient to one type of shock in the driver but less resilient to other types of shocks $[8,16]$. For example, tropical savanna are highly resilient to fires, but not to over grazing $[44,16,25]$; managed marine coral communities might be able to bounce back from small scale thermal or nutrient shocks faster by increasing engineering resilience, however, this would be at the risk of being wiped off by large scale shocks thereby decreasing ecological resilience [8].

Although these past studies provide some insight into resilience, particularly in response to isolated instantaneous shock in system drivers, a theoretical framework for understanding resilience in systems driven by continuously varying drivers represented as a stochastic process has not been developed. In this paper we develop a mathematical framework that provides important insights on different resilience measures and how they change with the attributes of the properties of the system and the driver variability. We hope that this development will also provide an interpretive framework for data driven investigations that use a probabilistic approach for the interpretation of the empirically observed dynamics $[45,33]$. We use the canonical catastrophic shift system to develop this framework to quantify resilience at aggregate spatial scales (spatially averaged) and stationary time scales (transient behavior is not considered) by arguing that different resilience attributes give rise to different measures [6, 23]. We provide insights by comparing the resilience of the system when subject to 
instantaneous shock and continuous variation in system drivers.

Developing on the work of past resilience quantifications $[7,16,55]$, we propose a system-driver-attribute triplet framework to quantify resilience measures. This triplet framework is best captured by asking the question: 'resilience of which behavioral characteristics, to what shock or variability in driver, and in what attribute'. Resilience 'of' can be system DOA or dynamic regime or other behavioral characteristics [30] which include function, process, feedbacks etc.; resilience 'to' can be the specific changes in the driver such as instantaneous shock or continuous variation in driver etc.; resilience 'in' can be distance to unstable threshold (ecological resilience), size of the DOA (width of the stability domain), mean dynamic regime residence time (fraction of time spent in the dynamic regime), mean passage times (switching frequency between adjoining connected dynamic regimes) etc. We also note that scale at which our resilience measures are proposed are important and in this study we deal with aggregate spatial scales and stationary time scales.

The primary goal of this paper is to define resilience measures for a dynamical system subject to continuous variation of drivers modeled as stochastic noise, and provide a comparison with those corresponding to instantaneous shock in drivers. We use catastrophic shift system as the canonical form of the model for illustration in this paper. Two ecological models are used as illustrations to provide context for comparing and contrasting these resilience measures. Out goal here is not to study these ecological systems in conventional detail, but to provide insights into the use of resilience measures as applied to these systems. In section 2, we develop the mathematical framework for the resilience measures in a catastrophic shift system subject to instantaneous shock, and continuous variation in system drivers characterized as (a) Gaussian white noise, and (b) Markovian dichotomous noise. Gaussian white noise captures random uncorrelated driver variability while Markovian dochotomous noise allows us to capture more structure in the driver variability. Using this mathematical framework, we develop resilience measures under instantaneous shock and continuous variability in system drivers (sections 3 and 4 respectively). We then analyze 
the characteristics of these resilience measures under varying system and driver parameters and highlight the emergent behavior. Subsequently, in section 5 we develop the concept of iso-resilience and in section 6 we provide discussions on the applications of our modeling approach. In section 7 we provide concluding remarks along with some broader implications of the results.

\section{Mathematical Formulations}

\subsection{Example A: Bistable population dynamics}

In this example we consider the dynamics of bistable populations with refuge and outbreak dynamic regimes subject to immigration and emigration, population growth, non-linear interspecific competitive effects and random stochastic variation in population fluxes. The governing equation that describe the dynamics of this population $P$ [number] at annual time scales is adapted from the logistic equation $[37,35]$ as:

$$
\frac{d P}{d t}=I+r P+c\left(\frac{P_{0}-P}{P_{0}}\right)^{k}+N \eta(t)
$$

The immigration and emigration rate $I$ [number/year] is a constant that can be positive or negative and population growth is governed by first order kinetics dependent on the intrinsic growth rate $r$ [1/year]. Interspecific competitive effects such as consumption, preemption, overgrowth, chemical interactions, territoriality and encounter competition etc. [46], are driven by a power law relationship dependent on: i) the population carrying capacity $P_{0}$ [number], ii) the strength of the competitive effects $c$ [number/year], and iii) the non-linearity of the competitive effects $k[-]$. The random variation effects such as inter-annual variations in immigration and emigration rates, mortality due to disease etc. are represented by a Gaussian white noise $\eta(t)$ [number/year] with standard deviation $N[-] . \quad k=3$ gives rise to catastrophic shift behavior. The range of admissible values for these parameters are: $P \geq 0, t>0,-\infty<I<\infty$, $r>0, c>0, P_{0}>0$ and $N \geq 0$. The population variable $P$ in equation (1) can 
be non-dimensionalized by using a relative population variable $x=\frac{P-P_{0}}{P_{0}}[-]$. Performing this substitution in equation (1) and simplifying for $k=3$ we get:

$$
\frac{d x}{d t}=\frac{I}{P_{0}}+r+r x-\frac{c}{P_{0}} x^{3}+\frac{N}{P_{0}} \eta(t)
$$

\subsection{Example B: Multi-stable vegetation dynamics}

The second example describes the dynamics of multi-stable vegetation cover dynamics with desert, intermediate and vegetated dynamic regimes subject to reforestation and deforestation, vegetation growth and colonization, competition, and random variations in vegetation mortality caused by stress and nonstress conditions induced by environmental fluctuations. The governing equation for the dynamics of vegetation as determined by its vegetation cover $F\left[\mathrm{~m}^{2}\right]$ at annual time scales is based on a simplified modeling framework presented by Ridolfi et al. [39] and is given as:

$$
\frac{d F}{d t}=A+g F+S\left(\frac{F_{0}-F}{F_{0}}\right)^{k}+N \eta(t)
$$

The deforestation and reforestation rate $A\left[\mathrm{~m}^{2} /\right.$ year $]$ is a constant that can be positive or negative and vegetation cover growth is governed by first order kinetics dependent on the intrinsic growth rate $g$ [1/year]. Competitive effects such as consumption, overgrowth, chemical interactions etc. [46], are driven by a power law relationship dependent on: i) the vegetation cover carrying capacity $F_{0}\left[m^{2}\right]$, ii) the strength of the competitive effects $S\left[\mathrm{~m}^{2} /\right.$ year $]$, and iii) the non-linearity of the competitive effects $k[-]$. The random fluctuation effects such as inter-annual variations in mortality rates are modeled as a Markovian dichotomous noise $\eta(t)\left[\mathrm{m}^{2} /\right.$ year $]$ with a variability intensity $N[-] . k=3$ gives rise to the catastrophic shift behavior. The range of admissible values for these parameters are: $F \geq 0, t>0,-\infty<A<\infty, g>0, S>0, F_{0}>0$ and $N \geq 0$. The vegetation cover variable $F$ in Equation (3) can be non-dimensionalized by using a normalized fractional vegetation variable $x=\frac{F-F_{0}}{F_{0}}[-]$. Performing this substitution in equation (3) and simplifying for $n=3$ we get:

$$
\frac{d x}{d t}=\frac{A}{F_{0}}+g+g x-\frac{S}{F_{0}} x^{3}+\frac{N}{F_{0}} \eta(t)
$$


These two systems will henceforth be referred to as Example A and Example $\mathrm{B}$ in the remainder of this paper. We note that, both equations (2) and (4) can be written in the generic form:

$$
\frac{d x}{d t}=\theta_{1}+\theta_{2} x+\theta_{3} x^{3}+K \eta(t)
$$

where, $\theta_{1}, \theta_{2}$ and $\theta_{3}$ are the parameters of the model and $K$ characterizes the strength of the noise. We note that in the absence of noise, i.e., $\eta(t)=0$, the dynamics corresponds to that of a double well potential. However, under noise, this behavior can be altered and the implications of this is explored in greater detail in this paper.

\subsection{Catastrophic shift system dynamics}

The governing equation for a general univariate deterministic dynamical system can be expressed as [4]:

$$
\frac{d x}{d t}=-\frac{d V}{d x}
$$

where, $x$ is the system state [in units of quantity $Q$ ], $t$ is time $[T]$ and $V(x)$ is the potential function $\left[Q^{2} / T\right]$. The canonical form for the dynamics describing a catastrophic shift system is [50]:

$$
\frac{d x}{d t}=\theta_{1}+\theta_{2} x+\theta_{3} x^{3} \equiv F(x)
$$

where $\theta_{1}, \theta_{2}$ and $\theta_{3}$ are the imperfection parameter (or flux parameter) $[Q / T]$, rate parameter (or growth/decay parameter) $[1 / T]$, and non-linear recovery parameter (or feedback parameter) $\left[1 /\left(Q^{2} T\right)\right]$, respectively. Comparing equation (2) (assuming $N=0$ ) with (7) we see that the flux parameter is the sum of the relative immigration and emigration rate and the relative population growth rate at the carrying capacity, i.e., $\theta_{1}=\frac{I}{P_{0}}+r[1 /$ year $]$, the rate parameter is the intrinsic population growth rate $\theta_{2}=r$ [1/year], and the feedback parameter is the normalized strength of competitive effects $\theta_{3}=\frac{-c}{P_{0}}[1 /$ year $]$. And comparing equation (4) (assuming $N=0$ ) with (7) we see that the flux parameter is the sum of the relative deforestation and reforestation rate and the normalized fractional vegetation growth rate at the carrying capacity i.e. $\theta_{1}=\frac{A}{F_{0}}+g[1 /$ year $]$, 
the rate parameter is the intrinsic vegetation cover growth rate $\theta_{2}=g[1 /$ year], and the feedback parameter is the normalized strength of competitive effects $\theta_{3}=\frac{-S}{F_{0}}[1 /$ year $]$.

The expression for the potential function $V$ in equation (7) is governed by $-\frac{d V}{d x}=\frac{d x}{d t}$ and is obtained as:

$$
V(x)=-\left(\theta_{1} x+\frac{\theta_{2} x^{2}}{2}+\frac{\theta_{3} x^{4}}{4}\right)
$$

Three cases are possible depending on the parameter combinations of $\theta$. Case 1: When $27 \theta_{1}^{2}<\frac{4 \theta_{2}^{3}}{\theta_{3}}$ three real fixed points are obtained. If $\theta_{3}<0$, then two of the three fixed points will be stable giving rise to the well known bi-stable system. For the rest of this paper, we will use equation (7) and without loss of generality assume $\theta_{3}=-1$. Case 2 : When $27 \theta_{1}^{2}=\frac{4 \theta_{2}^{3}}{\theta_{3}}$ two real fixed points are obtained, and Case 3: When $27 \theta_{1}^{2}>\frac{4 \theta_{2}^{3}}{\theta_{3}}$ one real fixed point is obtained.

We denote the $i^{\text {th }}$ stable fixed point, or attractor, as $\dot{x}_{i}$ and $X_{i}$ as its DOA (Fig. 1a). $\stackrel{\circ}{x}_{i, j}$ is the unstable (fixed point) boundary between the DOAs $X_{i}$ and $X_{j}$. DOA $X_{i}$ can be bounded by the unstable boundaries $\stackrel{\circ}{x}_{i, j}$ and $\stackrel{\circ}{x}_{i, k}$ or an externally imposed system boundary $\bar{x}_{i}$.

In Example A, when $N=0$, and under the condition $27 \theta_{1}^{2} \theta_{3}<4 \theta_{2}^{3}$, we have bistable population dynamics with the stable low relative population level $\dot{x}_{1}$ termed as 'refuge population' and the stable high relative population level $\dot{x}_{2}$ termed as 'outbreak population' [50]. The unstable relative population level $\stackrel{\circ}{x}_{1,2}$ separating the refuge and outbreak relative population levels is termed as 'threshold population' [50]. When the initial relative population is higher than the threshold population, the population eventually reaches the outbreak population, and when the initial relative population is lower than the threshold population, the population eventually reaches the refuge population (Fig. 1a). The two DOAs $X_{1}$ and $X_{2}$ are called 'refuge DOA' and 'outbreak DOA' and these correspond to the stable attractors $\dot{x}_{1}$ and $\dot{x}_{2}$ respectively.

Similarly, in Example B, when $N=0$, and under the condition $27 \theta_{1}^{2} \theta_{3}<4 \theta_{2}^{3}$, we have multi-stable vegetation cover dynamics with the stable low normalized fractional vegetation cover $\dot{x}_{1}$ termed as 'desert cover' and the stable high nor- 
malized fractional vegetation cover $\dot{x}_{2}$ termed as 'vegetated cover' [39, 44]. The unstable normalized fractional vegetation cover $\stackrel{\circ}{x}_{1,2}$ separating the desert and vegetated covers is termed as 'threshold cover'. When the initial normalized fractional vegetation cover is higher than the threshold cover, the ecosystem eventually reaches the vegetated cover, and when the initial normalized fractional vegetation cover is lower than the threshold cover, the ecosystem eventually reaches the desert cover (Fig. 1b). The two DOAs $X_{1}$ and $X_{2}$ are called 'desert DOA' and 'vegetated DOA' and these correspond to the stable attractors $\dot{x}_{1}$ and $\dot{x}_{2}$ respectively.

The fixed points for equation (7) can be obtained as:

$$
\begin{array}{r}
\dot{\bullet}_{1}=-\left(\frac{\sqrt{3 i}}{2}+\frac{1}{2}\right) a^{\frac{1}{3}}-\left(\frac{\sqrt{3 i}}{2}-\frac{1}{2}\right) \frac{\theta_{2}}{3 \theta_{3} a^{\frac{1}{3}}} \\
\stackrel{\circ}{x}_{1,2}=\left(\frac{\sqrt{3 i}}{2}-\frac{1}{2}\right) a^{\frac{1}{3}}+\left(\frac{\sqrt{3 i}}{2}+\frac{1}{2}\right) \frac{\theta_{2}}{3 \theta_{3} a^{\frac{1}{3}}} \\
\dot{x}_{2}=a^{\frac{1}{3}}-\frac{\theta_{2}}{3 \theta_{3} a^{\frac{1}{3}}}
\end{array}
$$

where, $a=\frac{1}{2 \theta_{3}}\left(3^{\frac{-3}{2}} \sqrt{\frac{27 \theta_{1}^{2} \theta_{3}+4 \theta_{2}^{3}}{\theta_{3}}-\theta_{1}}\right)$. Note that, while the expressions for the fixed points involve complex numbers $i=\sqrt{-1}$, these simplify under the required range of parameters to yield real fixed points.

\subsection{Catastrophic shift system dynamics under instantaneous shock or continu-} ous variability in drivers

In this paper, we consider two different types of changes in system drivers: (i) instantaneous shock and, (ii) continuous variability, where the effect of driver variability on the systems dynamics are tightly coupled. An instantaneous shock in the driver is characterized by the Dirac delta function where the shock is applied on a steady state initial condition at an instantaneous time point and the system state is allowed to relax in response to this shock eventually returning to one of the attractors. In Example A, the instantaneous shock can be an externally imposed shock (time scales much smaller than that of the population dynamics) in population in-flux or out-flux caused by sudden changes in 
immigration and emigration or rapid mortality events etc. In Example B, the instantaneous shock event can be a shock in vegetation cover in-flux or out-flux caused by extreme events such as rapid deforestation or reforestation, insect attack, forest fires etc.

The dynamics of a system when subject to instantaneous shock in drivers can be analyzed using the mathematics described in section 2.3. However, when the system given by equation (7) is driven by a continuously varying driver modeled using a stochastic additive noise, it can be expressed as [4]:

$$
\frac{d x}{d t}=\theta_{1}+\theta_{2} x+\theta_{3} x^{3}+\sqrt{2 D} \eta(t)
$$

where $\eta(t)$ is the noise term $[Q / T]$ and $D$ is its scaling factor [-]. If we consider noise with zero mean, then increasing $D$ results in increasing the variability of the driver. Note that the mean of the variation in the driver can be factored out and combined with the $\theta_{1}$ term, hence changes in the mean values of the noise can be represented as changes in $\theta_{1}$. We consider two different noise types, namely: Gaussian white noise and Markovian dichotomous noise. While Gaussian white noise captures uncorrelated variability, Markovian dichotomous noise is a switching process between two levels $\Delta_{1}$ or $\Delta_{2}[Q]$. The transition rates from $\Delta_{2}$ to $\Delta_{1}$, and from $\Delta_{1}$ to $\Delta_{2}$, are distributed as $\exp \left(\gamma_{1}\right)$ and $\exp \left(\gamma_{2}\right)$, respectively, where $\gamma_{1}$ and $\gamma_{2}$ are the mean switching frequencies $[1 / T]$. Note that $1 / \gamma_{1}$ and $1 / \gamma_{2}$ are the mean residence times in levels $\Delta_{2}$ and $\Delta_{1}$ respectively. These noise types allow us to capture changes in the intensity (by changing $\Delta_{1}$ and $\Delta_{2}$ ), variability (by changing $D$ ), frequency (by changing $\gamma_{1}$ and $\gamma_{2}$ ) and asymmetric bias (by making $\Delta_{1} \neq \Delta_{2}$ ) of driver variability.

Gaussian white noise has been used to model the fluctuations in mean nutrient loading in lakes or oceans [7], temporal variation in population reproduction rate and/or population carrying capacity [34, 39], variation in precipitation-soil moisture feedback strength [40], etc. Example applications of dichotomous noise include the switching between stress and non-stress conditions in resource availability [39], occurrence of fires in savanna [21], switching between wet and dry seasons in precipitation patterns such as drought versus no drought [39] etc. 
The resilience attributes of the system given by equation (10), driven by continuous variability in drivers, are best described in terms of the properties of the probability density function $(\mathrm{PDF}) P_{S}(x)$, of the state variable $x$, at the appropriate space and time scale of interest. In the context of a stochastically driven catastrophic system, the peaks or modes $\dot{x}_{i}$ of $P_{S}(x)$ represent locations of maximum probabilities of system state (most preferred state) whereas the troughs or anti-modes $\stackrel{\circ}{x}_{i, j}$ (between the modes $\dot{x}_{i}$ and $\dot{x}_{j}$ ) represent locations of minimum probabilities of system state (least preferred state). We define $X_{i}$ as the dynamic regime of the mode $\dot{x}_{i}$, bounded by anti-modes $\stackrel{\circ}{x}_{i, j}$ and $\stackrel{\circ}{x}_{i, k}$ or an externally imposed system boundary $\bar{x}_{i}$ (Fig. 1b).

The concept of dynamic regime in the presence of a continuously varying driver is analogous to the concept of DOA in the absence of an external driver. Note that, the restriction of $27 \theta_{1}^{2} \theta_{3}<4 \theta_{2}^{3}$ for the existence of multi-stability does not apply for the case of dichotomous noise. This is because, non-linear systems subject to noise can exhibit noise-induced bifurcation where the structural characteristics of the stationary PDFs change depending upon the characteristics and magnitude of the noise $[4,26,39]$. In Example A, we have two dynamic regimes $X_{1}$ and $X_{2}$ called 'refuge dynamic regime' and 'outbreak dynamic regime', and these correspond to the modes $\dot{x}_{1}$ and $\dot{x}_{2}$ respectively. Similarly, in Example B, depending on the value of the driver variability $\sqrt{2 D}$ we can have upto four dynamic regimes $X_{1}, X_{2}, X_{3}$ and $X_{4}$ called 'desert dynamic regime', 'intermediate desert dynamic regime', 'intermediate vegetated dynamic regime' and 'vegetated dynamic regime', and these correspond to the modes $\dot{x}_{1}, \dot{x}_{2}, \dot{x}_{3}$ and $\dot{x}_{4}$ respectively (see section 4.3 ).

\section{Catastrophic shift system subject to instantaneous shock}

\subsection{Resilience measures for Instantaneous shock in system drivers}

We now define the following resilience measures for a catastrophic shift system given by equation (7) subject to an instantaneous shock in system driver. A summary of these measures is provide in Table 1: 
- Size of DOA $\mathscr{R}_{i}^{s}=\left|\stackrel{\circ}{x}_{i, j}-\stackrel{\circ}{x}_{i, k}\right|[Q]$ : This measure captures the range of all possible values that the system state $x$ can take so that it will remain within the DOA at all future times. This measure has also been referred to as the latitude $[6,16,55]$. For the example illustrated in Fig. 1a, the two measures $\mathscr{R}_{1}^{s}$ and $\mathscr{R}_{2}^{s}$ are $\infty$, since one of the boundaries of each domain goes to $\pm \infty$. In general it can be said that larger the $\mathscr{R}_{i}^{s}$ the greater the allowance for the variation of $x$ where it will relax to $\dot{x}_{i}$ and hence greater the resilience. This is illustrated in Fig. 2c. However, this measure does not take into account the skewness of the location of the attractor $\dot{x}_{i}$ with respect to its boundaries. In Example A, the size of DOA measure $\mathscr{R}_{1}^{s}$ represents the extent of the refuge DOA. A large $\mathscr{R}_{1}^{s}$ generally requires a greater shock magnitude for the system to undergo a transition to the outbreak DOA. Similarly, in Example B, the size of the DOA measure $\mathscr{R}_{2}^{s}$ represents the extent of the vegetated DOA.

- Ecological resilience $\mathscr{R}_{i}^{E}=\min _{j}\left\{\left|\dot{x}_{i}-\stackrel{\circ}{x}_{i, j}\right|\right\} \quad[Q]$ : This measure captures how far the attractor of system state $\dot{x}_{i}$ is from the unstable DOA boundaries $\stackrel{\circ}{x}_{i, j}$ [18]. This measure has also been referred to as precariousness $[6,16,55]$. For the example illustrated in Fig. 1a, the two measures $\mathscr{R}_{1}^{E}$ and $\mathscr{R}_{2}^{E}$ are highlighted. The system state will be at the attractor at steady state, and is the preferred state of the DOA $X_{i}$. If the location of the attractor $\dot{x}_{i}$ is close to the unstable DOA boundary, then in terms of the difference in state values $x$, a smaller 'push' is sufficient to transition the system state into another DOA. Such a system is less resilient to DOA changes, and hence we say that it has a smaller ecological resilience. In Example A, the ecological resilience measure $\mathscr{R}_{2}^{E}$ represents the amount of 'push' in terms of shocks in immigration and emigration required for the population to undergo a transition from the outbreak population to the refuge population.

This measure takes into account the skewness associated with the locations of the outbreak and threshold population as demonstrated in Fig. 2a. In 
this example while the $\mathscr{R}_{i}^{s}$ measures are the same, the $\mathscr{R}_{i}^{E}$ measures are different. In Example A, if $\frac{\mathscr{R}_{1}^{E}}{\mathscr{R}_{2}^{E}}=2$ we can say that the refuge population levels are two times more resilient than the outbreak population levels against undergoing a transition (to the alternate stable population level) when subject to a shock in immigration and emigration rate. In Example B, the ecological resilience measure $\mathscr{R}_{1}^{E}$ represents the amount of 'push' (fraction of vegetation cover wiped out or planted) in terms of shocks in mortality required for the vegetation to undergo a transition from the desert cover to the vegetated cover.

- Engineering resilience $\mathscr{R}_{i}^{e}=\min _{j}\left\{\frac{1}{T_{i, j}}\right\} \quad[1 / T]$ : This measure captures the rate of recovery following a shock in the system driver, where $T_{i, j}$ denotes travel time from the unstable boundary $\stackrel{\circ}{x}_{i, j}$ to the attractor $\dot{x}_{i}$ caused by an infinitesimally small perturbation $\epsilon$ [24]. If a DOA $X_{i}$ has a large $T_{i, j}$, it means that the recovery towards the attractor is slow and correspondingly the $\mathscr{R}_{i}^{e}$ is low; and conversely a small $T_{i, j}$ results in a faster recovery and hence a higher $\mathscr{R}_{i}^{e}$. We note that typically the system moves very slowly near the fixed points and contributes most to the total travel time. The total travel time is therefore strongly dependent on the choice of $|\epsilon|$. In Example $A$, the engineering resilience measure $\mathscr{R}_{1}^{e}$ is the rate of recovery for the relative population to travel from the threshold level to the refuge level when subject to an infinitesimally small perturbation $\epsilon$. Similarly, in Example B, the engineering resilience measure $\mathscr{R}_{2}^{e}$ is the rate of recovery for the normalized fractional vegetation cover to recover from the threshold to the vegetated cover when subject to an infinitesimally small perturbation $\epsilon$.

The travel time $T_{i, j}$ from the unstable boundary $\stackrel{\circ}{x}_{i, j}$ to the attractor $\dot{x}_{i}$ when subject to an $\epsilon$ perturbation is computed using the following expression:

$$
T_{i, j}=\int_{\substack{\dot{\circ}_{i, j}+\epsilon \\ x_{i}-\epsilon}}^{\dot{\bullet}_{i}} \frac{d x}{F(x)}
$$


For the illustration discussed later in section 3.2, the parameter $\epsilon$ is set as $0.01 \%$ of $\left|\stackrel{\circ}{x}_{i, j}-\dot{x}_{i}\right|$. While the choice of $\epsilon$ affects the actual numerical values of $T_{i, j}$, the trends in $T_{i, j}$ across a range of parameters remain unaffected. Substitution of $\dot{x}_{1}, \dot{x}_{2}$ and $\stackrel{\circ}{x}_{1,2}$ values from equation (9) in (11) and integrating numerically using the trapezoid method gives us the $T_{i, j}$ values. $\mathscr{R}_{i}^{e}$ is readily obtained as the inverse of $T_{i, j}$.

- Attractor response rate $\mathscr{R}_{i}^{\lambda}=\left|\frac{d F(x)}{d x}\right|_{x=\dot{x}_{i}}[1 / T]$ : This measure provides an alternate estimate for the rate of recovery following a shock in the system driver in terms of the inverse of the characteristic time $\tau_{i}=\left|\frac{d F(x)}{d x}\right|_{x=\dot{x}_{i}}^{-1}$ when subject to a perturbation at its attractor $\dot{x}_{i}$ [39]. The function $\left|\frac{d F(x)}{d x}\right|$ can be computed from equation (7) as:

$$
\left|\frac{d F(x)}{d x}\right|=\left|\theta_{2}+3 \theta_{3} x^{2}\right|
$$

$\mathscr{R}_{i}^{\lambda}$ is obtained by direct substitution of the values of $\dot{x}_{1}$ and $\dot{x}_{2}$ from equation (9) in equation (12). A DOA with a small $\mathscr{R}_{i}^{\lambda}$ indicates that the system converges to its stable state at a slower rate as compared to another DOA with a larger $\mathscr{R}_{i}^{\lambda}$. Unlike $\mathscr{R}_{i}^{e}$ which involves some subjectivity in the choice of $\epsilon, \mathscr{R}_{i}^{\lambda}$ presents a more attractive alternative to capture this aspect of the resilience. However, it must be noted that, while $\mathscr{R}_{i}^{\lambda}$ provides an indication of the recovery time only near the attractor, $\mathscr{R}_{i}^{e}$ includes the entire DOA. This is illustrated in Fig. 2 b and d. In Example A, $\mathscr{R}_{2}^{\lambda}$ measures how fast the relative population recovers to the outbreak population level when subject to minor shocks. Similarly, in Example B, $\mathscr{R}_{1}^{\lambda}$ measures how fast the normalized fractional vegetation cover recovers to the desert cover level when subject to minor shocks. Note that while $\mathscr{R}_{1}^{e}$ measures the recovery rate from the unstable threshold cover to the stable desert cover, $\mathscr{R}_{1}^{\lambda}$ only measures the recovery rate near the stable desert cover.

- Activation potential $\mathscr{R}_{i}^{a}=\min _{j}\left\{V\left(\stackrel{\circ}{x}_{i, j}\right)-V\left(\stackrel{\bullet}{x}_{i}\right)\right\} \quad\left[Q^{2} / T\right]$ : This measures the energy needed to transition the system from its attractor to one 
of its unstable boundaries. This measure is related to the definition of resistance $[6,16,55]$. For the example illustrated in Fig. 1a, the two measures $\mathscr{R}_{1}^{a}$ and $\mathscr{R}_{2}^{a}$ are highlighted. While the ecological resilience measure $\mathscr{R}_{i}^{E}$ deals with the 'push' in terms of the difference in state values, the activation potential measure deals with the 'push' in terms of energy. This difference is highlighted in Fig. 2c. Using the potential function we can compute the minimum energy (potential) required to transition the system state from its attractor $\dot{x}_{i}$ to its boundary $\stackrel{\circ}{x}_{i, j}$. The lower the energy required to achieve this, the easier it is to accomplish the transition, and hence the lower the activation potential resilience. $\mathscr{R}_{i}^{a}$ is easily computed by substitution of $\dot{x}_{1}, \dot{x}_{2}$ and $\stackrel{\circ}{x}_{1,2}$ values from equation (9) in equation (8). In Example A, the activation potential measure $\mathscr{R}_{1}^{a}$ measures the energy (in terms of relative population potential) needed to transition from a refuge population to an outbreak population. And in Example B, the activation potential measure $\mathscr{R}_{2}^{a}$ measures the energy (in terms of normalized fractional vegetation cover potential) needed to transition from a vegetated cover to a desert cover.

While this is not an exhaustive list of resilience measures, we believe that it includes measures that would be encountered quite frequently across a wide spectrum of problems. Furthermore, these measures captures the key differences in the dynamics of system behavior as illustrated in Fig. 2. It is also possible to obtain derived resilience measures such as state recovery velocity $\mathscr{R}_{i}^{E v}=\mathscr{R}_{i}^{E} \mathscr{R}_{i}^{e}$ and activation potential recovery velocity $\mathscr{R}_{i}^{a v}=\mathscr{R}_{i}^{a} \mathscr{R}_{i}^{e}$ from the measures presented above. We now perform example simulations and analyze the behavior of these resilience measures under changing parameter conditions.

\subsection{Resilience behavior under instantaneous shock in system driver}

For the system described by (7) with $-\infty<\dot{x}_{1}<\stackrel{\circ}{x}_{1,2}<\dot{x}_{2}<\infty$ (in the allowable region determined by $27 \theta_{1}^{2} \theta_{3}<4 \theta_{2}^{3}$ ), the DOAs $X_{1}$ and $X_{2}$ are semi-infinite and therefore $\mathscr{R}_{1}^{s}=\mathscr{R}_{2}^{s}=\infty$. The variations of $\mathscr{R}_{i}^{E}, \mathscr{R}_{i}^{a}, \mathscr{R}_{i}^{e}$ 
and $\mathscr{R}_{i}^{\lambda}$ as a function of the variation in the imperfection parameter $\theta_{1}$ and the rate parameter $\theta_{2}$ are shown in Fig. 3. It is immediately evident that for the two DOAs, the four resilience measures show different variations as the parameters change. In the context of the ecological resilience, $\mathscr{R}_{1}^{E}$ decreases while $\mathscr{R}_{2}^{E}$ increases as the imperfection parameter $\theta_{1}$ increases (Fig. 3a). This is because as $\theta_{1}$ is increased, the distance between $\dot{x}_{1}$ and $\stackrel{\circ}{x}_{1,2}$ becomes larger and the distance between $\dot{x}_{2}$ and $\stackrel{\circ}{x}_{1,2}$ becomes smaller. Unlike $\theta_{1}$, the effect of increasing $\theta_{2}$ is to increase both $\mathscr{R}_{1}^{E}$ and $\mathscr{R}_{2}^{E}$. This is because $\theta_{2}$ has the effect of pushing both the attractors $\dot{x}_{1}$ and $\dot{x}_{2}$ away from the unstable boundary $\stackrel{\circ}{x}_{1,2}$ irrespective of the nature of the imperfection. Hence, the highest ecological resilience measures $\mathscr{R}_{i}^{E}$ for a given DOA $X_{i}$ are obtained when we have high values of $\theta_{2}$, while the effect of increasing $\theta_{1}$ is dependent on the DOA under consideration. In Example B, high reforestation rate along with high vegetation growth rate increases the $\mathscr{R}^{E}$ of the vegetated DOA and decreases the $\mathscr{R}^{E}$ of the desert DOA.

The activation potential measure $\mathscr{R}_{i}^{a}$ shows a pattern similar to that of $\mathscr{R}_{i}^{E}$ although the gradients of change are quite different (Fig. 3b). This is because there is a one to one mapping between the distance and the potential for our particular problem (equation (8)). However, it must be noted that in general this behavior need not be true for other systems. In Example A, high emigration rates and low population growth rates increases the $\mathscr{R}^{a}$ of the refuge population and decreases the $\mathscr{R}^{a}$ of the outbreak population.

The engineering resilience measure $\mathscr{R}_{i}^{e}$ (Fig. 3c) shows patterns of variation that are distinctly different from those of $\mathscr{R}_{i}^{E}, \mathscr{R}_{i}^{a}$ and $\mathscr{R}_{i}^{\lambda}$ (Fig. $3 \mathrm{a}, \mathrm{b}$ and d). We see that as $\theta_{2}$ increases, the travel time decreases in both DOAs. Although $\theta_{2}$ increases the distance between the attractor and unstable boundary, it also significantly increases the travel velocity between these points (by making the slope steeper), with the net effect of decreasing travel time. The behavior of $\mathscr{R}_{i}^{e}$ with respect to $\theta_{1}$ is more complex. Maximum values of $\mathscr{R}_{i}^{e}$ are obtained at low $\left|\theta_{1}\right|$ values indicating that a symmetric system has the most travel time. When imperfection is introduced into the system $\left(\theta_{1} \neq 0\right)$, although the distance 
between the attractor and unstable boundary changes, there is a corresponding change in the travel velocity. The net effect is such that when distance increases the velocity decreases and vice versa. While larger $\left|\theta_{1}\right|$ results in increasing $\mathscr{R}_{i}^{E}$ on one DOA and decreasing $\mathscr{R}_{i}^{E}$ on the other DOA (Fig. 3a), $\mathscr{R}_{i}^{e}$ decreases with increasing $\left|\theta_{1}\right|$.

The behavior of the attractor response rate measure $\mathscr{R}_{i}^{\lambda}$ (Fig. $3 \mathrm{~d}$ ) to changes in $\theta_{1}$ and $\theta_{2}$ are similar to the $\mathscr{R}_{i}^{E}$ and $\mathscr{R}_{i}^{a}$ measures (Fig. 3 a and b) for identical reasons. In Example $\mathrm{B}$, high vegetation growth rates increases $\mathscr{R}_{i}^{\lambda}$ for both the desert and vegetated cover. While reforestation increases $\mathscr{R}_{i}^{\lambda}$ in vegetated cover and decreases $\mathscr{R}_{i}^{\lambda}$ in desert cover.

The above simulations illustrate the importance that attribute, or the choice of resilience measure, plays in determining our assessment of resilience under changing parameter conditions when the system is subject to an instantaneous shock. In the following section, we illustrate the resilience characteristics of a catastrophic shift system when subject to continuous variability in driver.

\section{Catastrophic shift system subject to continuous variability in drivers}

\subsection{Resilience measures for continuous variability in drivers}

We now define resilience measures for the stochastic dynamic system given by equation (10) and describe their properties under continuous variability in system drivers.

- Regime size $\mathscr{R}_{i}^{r}=\left|\stackrel{\circ}{x}_{i, j}-\stackrel{\circ}{x}_{i, k}\right|[Q]$ : This measure describes the extent of variability that the system state $x$ can take before it transitions out of dynamic regime $X_{i}$. For the example illustrated in Fig. 1b, the two measures $\mathscr{R}_{1}^{r}$ and $\mathscr{R}_{2}^{r}$ are $\infty$. A dynamic regime having a large size allows for greater variability in the system state while staying in the same dynamic regime and hence has greater $\mathscr{R}_{i}^{r}$ resilience. Note that unlike the concept of DOA, where the system state cannot alternate between DOAs, the concept of dynamic regimes allows the system state to alternate between connected dynamic regimes. Fig. 2 e and $g$ illustrates two dynamic 
regimes with equal and unequal regime size measures, respectively. In Example A, the regime size measure $\mathscr{R}_{1}^{r}$ represents the size of the refuge dynamic regime. In Example B, the regime size measure $\mathscr{R}_{2}^{r}$ represents the size of the vegetated dynamic regime.

- Regime probability $\mathscr{R}_{i}^{R}=\int_{X_{i}} P_{S}(x) d x[-]$ : This measure describes the probability of finding the system state $x$ in the dynamic regime $X_{i}$ for an ensemble of observations. For the example illustrated in Fig. 1b, the two measures $\mathscr{R}_{1}^{R}$ and $\mathscr{R}_{2}^{R}$ are the areas under their corresponding stationary PDFs. Using ergodic property (for connected dynamic regimes), this measure can also be interpreted as the average fraction of time that a system state stays in dynamic regime $X_{i}[26]$. Hence a dynamic regime with a higher $\mathscr{R}_{i}^{R}$ is more likely to be realized than a dynamic regime with a lower $\mathscr{R}_{i}^{R}$. Fig. 2 e and f illustrates two dynamic regimes with unequal and equal regime probabilities, respectively. In Example A, the dynamic regime probability measure $\mathscr{R}_{2}^{R}$ captures the average fraction of time the relative population is in the outbreak dynamic regime. Similarly, in Example $\mathrm{B}$, the regime probability measure $\mathscr{R}_{1}^{R}$ captures the average fraction of time the normalized fractional vegetation cover is in the desert dynamic regime. However, this is only true for the case of connected dynamic regimes. When we have disconnected dynamic regimes at barrier regions (as illustrated in section 4.3), the regime probability needs to be normalized with the sum of regime probabilities of connected dynamic regimes to obtain the average time fraction.

- Stochastic ecological resilience $\mathscr{R}_{i}^{s E}=\min _{j}\left\{\left|\stackrel{\bullet}{x}_{i}-\stackrel{\circ}{x}_{i, j}\right|\right\} \quad[Q]$ : This measure is similar to the ecological resilience measure $\mathscr{R}_{i}^{E}$, however it deals with the 'push' in terms of the difference in state values between the dynamic regime mode $\stackrel{\bullet}{x}_{i}$ and the boundary $\stackrel{\circ}{x}_{i, j}$. For the example illustrated in Fig. 1b, the two measures $\mathscr{R}_{1}^{s E}$ and $\mathscr{R}_{2}^{s E}$ are highlighted. Unlike $\mathscr{R}_{i}^{E}$, which indicates the exact amount of 'push' required to perform one DOA transition, $\mathscr{R}_{i}^{s E}$ only provides an indication of the amount of 'push' re- 
quired to have a tendency for dynamic regime transitions. This is because, under a stochastic variation in system drivers, dynamic regime changes occur infinitely often in the true limiting case of stationarity. Hence, a higher $\mathscr{R}_{i}^{s E}$ indicates that a larger 'push' in terms of differences in state values is required to undergo a tendency for dynamic regime changes. Fig. 2 f illustrates two dynamic regimes with equal $\mathscr{R}_{i}^{r}$, but unequal $\mathscr{R}_{i}^{s E}$ due to the skewness in the location of the mode with respect to the boundaries of the dynamic regime. In Example A, the stochastic ecological resilience measure $\mathscr{R}_{1}^{s E}$ represents the amount of 'push' in terms of shocks in immigration and emigration required for the population to undergo a tendency to transition from the refuge population to the outbreak population. Similarly, in Example B, the stochastic ecological resilience measure $\mathscr{R}_{2}^{s E}$ represents the amount of 'push' in terms of shocks in mortality required for the vegetation cover to undergo a tendency to transition from the vegetated cover to the neighboring intermediate or desert cover.

- Mean passage time $\mathscr{R}_{i}^{\tau}=\min _{j}\left\{\tau_{i, j}\right\} \quad[T]$ : This measure determines the minimum of the average time $\tau_{i, j}$ taken for a system in mode $\dot{x}_{i}$ of dynamic regime $X_{i}$ to transition to one of its neighboring mode $\dot{x}_{j}$ in dynamic regime $X_{j}$ [39]. A higher mean passage time causes the system to stay on an average longer in the present dynamic regime and hence results in a higher resilience. The inverse of the mean passage time gives the mean rate of transition between dynamic regime modes. Note that, the concept of $\mathscr{R}_{i}^{\tau}$ is different from $\mathscr{R}_{i}^{R}$ because two adjoining dynamic regimes $X_{i}$ and $X_{j}$ having identical residence times (equal regime probabilities) can accomplish this either by frequent or by rare transitions (small or large passage times) between them. In Example A, the mean passage time measure $\mathscr{R}_{2}^{\tau}$ represents the average time taken for the relative population to transition from the outbreak population to the refuge population levels. Similarly, in Example B, the mean passage time measure $\mathscr{R}_{1}^{\tau}$ represents the average time taken for the normalized fractional vegetation 
cover to transition from the desert cover to the neighboring intermediate or vegetated cover levels.

We now discuss the behavior of these resilience measures for the two different noise types.

4.2. Resilience behavior under driver variability modeled as Gaussian white noise

For the case of $\eta(t)$ being a Gaussian white noise, the stationary PDF for equation (10) is (see page 111, equation 6.10 or 6.13 in [26]):

$$
P_{S}(x)=N \exp \left\{\frac{-V(x)}{D}\right\}
$$

where $N=1 / \int_{X} \exp \left\{\frac{-V(x)}{D}\right\} d x$ is the normalizing factor computed numerically using trapezoid method, and $X$ represents the entire region where the stationary PDF exists. Note that both Ito and Stratanovich formulations yield the same result under additive Gaussian white noise variability in system drivers. The modes $\dot{x}_{i}$ and the antimodes $\stackrel{\circ}{x}_{i, j}$ are the locations of the local maxima and minima of the stationary PDF and are computed numerically. The regions between two consecutive local minima $\stackrel{\circ}{x}_{i, j}$ and $\stackrel{\circ}{x}_{i, k}$ or one local minima $\stackrel{\circ}{x}_{i, j}$ and one adjacent system boundary $\bar{x}_{i}$, or two system boundaries $\bar{x}_{i 1}$ and $\bar{x}_{i 2}$ (without any local minima between them) form dynamic regime $X_{i}$. For a catastrophic shift system driven by additive Gaussian white noise variability, when $27 \theta_{1}^{2}<\frac{4 \theta_{2}^{3}}{\theta_{3}}$ and $D<D_{t}$, we have two dynamic regimes $X_{1}$ and $X_{2}$ coinciding with the two DOAs, with the locations of the modes coinciding with the stable fixed points $\dot{x}_{1}$ and $\dot{x}_{2}$ (for the deterministic system) and the locations of the anti-mode coinciding with the unstable fixed point $\stackrel{\circ}{x}_{1,2}$ (for the deterministic system) (Fig. 4b). The modes have sharper peaks at low values of $D$ and they gradually diffuse as $D$ increases (Fig. $4 \mathrm{a}$ ). Note that, $D_{t}$ is a threshold variability scaling parameter, beyond which the driver noise overshadows the dynamics governed by the potential function and the bimodal behavior of the stationary PDF is lost. In this study, we will only consider the case of $D<D_{t}$. 
$\mathscr{R}_{i}^{r}$ and $\mathscr{R}_{i}^{s E}$ are computed from the locations of the boundaries and the modes of dynamic regime $X_{i} . \mathscr{R}_{i}^{R}$ is computed by integrating the stationary PDF given by equation (13) between the boundaries of dynamic regime $X_{i}$ numerically using the trapezoid method.

The mean passage time $\tau_{i, j}$ for transitions from mode $\dot{x}_{i}$ to mode $\dot{x}_{j}$ is (see page 81 , equation 3.60 in [39]):

$$
\tau_{i, j}=\frac{1}{D} \int_{\dot{x}_{1}}^{\dot{x}_{2}}\left[\exp \left\{\frac{V(x)}{D}\right\} \int_{-\infty}^{x} \exp \left\{\frac{-V(y)}{D}\right\} d y\right] d x
$$

The integrals in equation (14) are evaluated numerically using the trapezoid method. Note that, $\mathscr{R}_{i}^{\tau}=\min _{j}\left\{\tau_{i, j}\right\}$ and can be directly obtained from the above computation. We now perform example simulations and analyze the behavior of these continuous resilience measures under changing variability intensity and parameter conditions.

Increasing $D$ has the effect of destabilizing the more stable dynamic regime, and since $\sum_{i} \mathscr{R}_{i}^{R}=1$, the less stable dynamic regime tends to gain more regime probability. Hence an increase in the Gaussian white noise intensity $D$, (and correspondingly the driver variability), favors the otherwise less resilient dynamic regime, as measured by $\mathscr{R}_{i}^{R}$, at the cost of the more resilient dynamic regime (Fig. 4c). This variability induced stabilization of less resilient dynamic regimes, and consequently the destabilization of more resilient dynamic regimes, can have significant consequences in several ecological applications. One example is the stabilization of tropical savanna by fire induced variability [21]. In the absence of such a such fires, savanna become less resilient in terms of $\mathscr{R}_{i}^{R}$ and can potentially lead to shrub or woody encroachment.

Different parameter combinations for $\theta_{i}$ can give rise to different 'regions of sensitivity' where $\mathscr{R}_{i}^{R}$ changes more rapidly in response to changes in $D$ (indicated by regions around the highest slopes $\left|\frac{d \mathscr{R}_{i}^{R}}{d D}\right|$ in Fig. 4c). The range of values of $D$ for which $\mathscr{R}_{i}^{R}$ is most sensitive represents a critical region in terms of determining sensitivity to dynamic regime dominance. Some parameter combinations exhibit behavior where increasing variability on a lightly varying environmental 
significantly alters its $\mathscr{R}_{i}^{R}$ resilience measure whereas increasing variability on a highly varying environment may not cause any significant change in $\mathscr{R}_{i}^{R}$. These types of behavioral response can have important consequences especially in the context of climate change induced ecosystem transitions [8, 16, 39] caused by increased variability in climate forcings [48]. For example, Knapp et al. [29] demonstrate that increasing rainfall variability in grassland ecosytems resulted in decreased above ground net primary productivity and increased plant community diversity or species richness, while D'Odorico and Bhattachan [11] show that increasing hydrologic variability can enhance post-shock recovery (engineering resilience or mean passage time measures) in dryland ecosystems.

The behavior of the system state as a function of time for different values of $D$ is illustrated in Fig. 5. It can be observed that when $D$ is increased, the frequency of switching between the two dynamic regimes increases. Furthermore, from the phase plots (Fig. 5b) it can be inferred that the system state tends to stay closer to the modes at low values of $D$, and there is an increased diffusion in the location of the system state as $D$ increases. This corresponds to a diffusion in the peaks of the stationary PDF as $D$ increases (Fig. 4).

The regime probability measure $\mathscr{R}_{i}^{R}$ is highly sensitive to changes in $\theta_{1}$ (Fig. 6a). In contrast, for a fixed $\theta_{1}$, variations in $\theta_{2}$ have significantly smaller change in $\mathscr{R}_{i}^{R}$. In a bimodal system, these variations are complementary between the two dynamic regimes. As we increase the driver variability $D$, we observe greater diffusion in the probability densities and this causes the regime probabilities $\mathscr{R}_{1}^{R}$ and $\mathscr{R}_{2}^{R}$ to get closer to each other and eventually become equal in the limiting case of large noise levels. Interpreting this in the context of Example A, we infer that the fraction of time spent in the refuge dynamic regime and the outbreak dynamic regime are highly sensitive to changes in immigration and emigration rates, with the sensitivity decreasing with increasing driver variability.

Similar to $\mathscr{R}_{i}^{R}, \mathscr{R}_{i}^{s E}$ is highly sensitive to $\theta_{1}$ for identical reasons (Fig. 6b). $\theta_{2}$ on the other hand has a mild effect of increasing $\mathscr{R}_{i}^{s E}$ irrespective of the dynamic regime (Fig. 6b). This is attributed to the fact that $\theta_{2}$ causes a stabilizing effect as mentioned earlier. Also, changing the driver variability $D$ 
does not affect $\mathscr{R}_{i}^{s E}$. This is because, for our particular problem the modes occur at the attractors $\dot{x}_{1}$ and $\dot{x}_{2}$ and the anti-mode occurs at the DOA boundary $\stackrel{\circ}{x}_{1,2}$ irrespective of the driver variability $D$ resulting in $\mathscr{R}_{i}^{s E}=\mathscr{R}_{i}^{E}$. However, it must be noted that in general this need not be true for other systems where the behavior of $\mathscr{R}_{i}^{s E}$ can be affected by $D$.

The mean passage time measure $\mathscr{R}_{i}^{\tau}$ increases with increasing $\theta_{2}$ (Fig. 6c) due to the stabilizing effect of $\theta_{2}$. $\mathscr{R}_{1}^{\tau}$ decreases while $\mathscr{R}_{2}^{\tau}$ increases as the imperfection parameter $\theta_{1}$ is increased. This is because, when $\theta_{1}$ is increased, the distance between $\dot{x}_{1}$ and $\stackrel{\circ}{x}_{1,2}$ becomes larger and the distance between $\dot{x}_{2}$ and $\stackrel{\circ}{x}_{1,2}$ becomes smaller, and the probability peak at $\dot{x}_{1}$ increases while the peak at $\dot{x}_{2}$ decreases. This effect is weakened with increasing driver variability since variability facilitates dynamic regime changes. The effect of driver variability is such that at $\theta_{1}=0.0[Q / T]$ doubling $\theta_{2}$ from 1.0 to $2.0[1 / T]$ results in 32 orders of magnitude increase in $\mathscr{R}_{1}^{\tau}$ at $D=0.01[-]$ but only a 3 orders of magnitude increase at $D=1.0[-]$ (Fig. 6c). Applying this to Example A, we infer that the mean passage time $\mathscr{R}_{1}^{\tau}$ decreases while $\mathscr{R}_{2}^{\tau}$ increases with increasing population growth rate. This means that on an average transitions from the outbreak to the refuge population levels occur less frequently as the population growth rate is increased. Also by increasing the immigration rate we can make the transition from the refuge to the outbreak population levels faster while simultaneously making the transition from the outbreak to the refuge population level slower.

4.3. Resilience behavior under driver variability modeled as Markovian dichotomous noise

For the case when $\eta(t)$ is a Markovian dichotomous noise, the stationary PDF for equation (10) is (see page 267, equation 9.50 in Horsthemke and Lefever [26]):

$$
P_{S}(x)=N^{\prime} \frac{\sqrt{2 D}}{\kappa(x)} \exp \left\{-\left(\gamma_{1}+\gamma_{2}\right) \int^{x} \frac{F(z)+I \sqrt{2 D})}{\kappa(z)} d z\right\}
$$

where $\kappa(x)=\left\{F(x)+\sqrt{2 D} \Delta_{1}\right\}\left\{\left(F(x)+\sqrt{2 D} \Delta_{2}\right\}, I=\frac{\gamma_{1} \Delta_{1}+\gamma_{2} \Delta_{2}}{\gamma_{1}+\gamma_{2}}, N^{\prime}\right.$ is a normalizing factor computed numerically using the trapezoid method. The integral in equation (15) is also performed numerically using the trapezoid method. 
The solution boundaries for equation (15) are computed as follows [39]. First, we compute the locations of fixed points for equation (10), by substituting the noise term $\eta(t)$ as $\Delta_{1}$ and $\Delta_{2}$. Combining the fixed points for these two cases, we look for regions where there are two consecutive stable fixed points. These regions form the valid solution boundaries for the stationary PDF. For the case when we have disconnected solution boundaries (barrier regions), we normalize each solution separately and finally scale all the stationary PDFs by the number of disconnected solutions. In some cases these solution boundaries can be points of singularity in which case we perform the numerical integration by introducing appropriate shifts from the boundaries.

The modes $\stackrel{\dot{x}}{i}_{i}$ and the antimodes $\stackrel{\circ}{x}_{i, j}$ are the locations of the local maxima and minima of the stationary PDF and are computed numerically. The regions between two consecutive local minima $\stackrel{\circ}{x}_{i, j}$ and $\stackrel{\circ}{x}_{i, k}$ or one local minima $\stackrel{\circ}{x}_{i, j}$ and one adjacent system boundary $\bar{x}_{i}$, or two system boundaries $\bar{x}_{i 1}$ and $\bar{x}_{i 2}$ (without any local minima between them) form dynamic regime $X_{i}$. For a catastrophic shift system driven by additive Markovian dichotomous noise, we can have anywhere between one and four dynamic regimes depending on the parameter values. These are caused due to noise induced bifurcations [26, 39]. Qualitative changes in dynamics of a system when subject to parameter change are called bifurcations. Bifurcations arising due to change in intrinsic system parameters (in our case $\theta_{1}, \theta_{3}$ and $\theta_{3}$ ) are regular bifurcations. For the example considered in this study, we deal with catastrophic bifurcations. However, bifurcations can also occur when we change the characteristics of the noise in a noise-driven dynamical system. These bifurcations are referred to as noise induced bifurcations. For the case of Markovian Dichotomous noise, which has four intrinsic noise parameters $\Delta_{1}, \Delta_{2}, \gamma_{1}$ and $\gamma_{2}$ which can vary independent of one other, we can induce bifurcations corresponding to the variation in each of these noise parameters. When bifurcations are induced due to changes in $\Delta$, they are referred to as ' $\Delta$ bifurcations' and when bifurcations are induced due to changes in $\gamma$, they are referred to as ' $\gamma$ bifurcations'.

$\mathscr{R}_{i}^{r}$ and $\mathscr{R}_{i}^{s E}$ are computed from the locations of the boundaries and the 
modes of dynamic regime $X_{i} . \mathscr{R}_{i}^{R}$ is computed by integrating the stationary PDF given by equation (13) between the boundaries of dynamic regime $X_{i}$ numerically using the trapezoid method. When we have disconnected dynamic regimes, as is the case with barrier regions indicated by the ellipse in Fig. 7b, we normalize each of the individual PDFs separately and then scale them by the number of disconnected regions.

The mean passage time $\tau_{i, j}$ for transitions from mode $\dot{x}_{i}$ to mode $\dot{x}_{j}$ is (see page 3524, equation 20 in Sancho [41]):

$$
\tau_{i, j}=\left(\gamma_{1}+\gamma_{2}\right) \int_{\dot{x}_{1}}^{\dot{x}_{2}}\left[\frac{-1}{\psi(x) \kappa(x)} \int_{\bar{x}_{i}}^{x} \psi(y) d y\right] d x
$$

where $\psi(x)=\frac{\left|\Delta_{1}\right| \sqrt{2 D}-F(x)}{\sqrt{2 D}} P_{S}(x)$. Equation (16) is evaluated numerically using the trapezoid method. Note that, $\mathscr{R}_{i}^{\tau}=\min _{j}\left\{\tau_{i, j}\right\}$ and can be directly obtained from the above computation. We now perform example simulations and analyze the behavior of these continuous resilience measures under changing noise properties and parameter conditions.

Unlike for the case of Gaussian white noise, when a catastrophic shift system is subject to a Markovian dichotomous noise variability in system drivers, we see structural changes in the stationary probability densities as the variability is increased (Fig. 7 a and d). These structural changes arise due to noise induced bifurcations. Distinction must be made between two regions which have four dynamic regimes each. For the example presented in Fig. 7b, this occurs between $0.0<D<0.0545$ which represents disconnected dynamic regimes and between $0.0705<D<0.0935$ which represents connected dynamic regimes. While the system state is able to explore a continuous domain in the region of connected dynamic regimes, in the region of disconnected dynamic regimes, there exists a dynamical barrier that prevents the system state from transitioning between the disconnected dynamic regimes. We refer to these regions where the system states are disconnected as barrier regions (Fig. 7b, $0.0<D<0.0545$ ). These dynamical barrier regions are illustrated through the use of time plots of the 
system state for different values of $D$ (Fig. 8). It can be observed from the figure that for $D=0.01$ and $D=0.04$, the system state stays around its corresponding higher and lower initial values $(X(0)=1.005$ and $X(0)=-0.995$ respectively) at all future times and hence the two regimes are disconnected. This behavior is true for $0<D<0.0545$. However, at $D=0.07$, when the system state starts at a higher initial value $(X(0)=1.005)$, it eventually reaches the dynamic regimes with lower values (around $X(0)=-0.995$ ). This illustrates the fact that the system state permanently escapes into the regimes with lower state values. This behavior is true for $0.0545<D<0.0705$. However, at $D=0.1$, the system state is able to move between all three dynamic regimes indicating that these are connected (Fig. 8). This behavior is true for $0.0705<D$.

The existence of barrier regions coupled with the phenomenon of noise induced bifurcations can lead to the creation of trap and escape regions as indicated by the ellipse in Fig. 7b. For the set of parameter combinations represented by case 1 in Fig. 7, when a system state starts in this region (indicated by the ellipse) and the driver variability varies between $0.0<D<0.0545$, the system state will remain trapped in this region at all future times. However, once the driver variability is scaled up to $D>0.0545$, the system state permanently escapes out of this region. Unlike deterministic catastrophic shift systems which allow for the recovery of original state with hysteresis through parameter change, this region can never be recovered by decreasing the driver variability. The existence of these trap or escape regions can have important consequences in the context of climate change induced irrecoverable ecosystem transitions caused by increased variability in climate forcings [39]. This is because, we can have catastrophic losses to ecosystem processes and function just by changing the variability in the climate, and more importantly the losses are irrecoverable even when the climate variability is decreased.

It must be noted that, for the case of Markovian dichotomous noise, we have four parameters $\Delta_{1}, \Delta_{2}, \gamma_{1}$ and $\gamma_{2}$. By varying each of these parameters independently, we can obtain noise induced bifurcations at four possible locations as illustrated in Fig. 7b. It can be observed from Fig. $7 \mathrm{~b}$ and c that, while both 
$\Delta$ bifurcations change the number of dynamic regimes, only one $\gamma$ bifurcation occurring at $D=0.0935$ changes the number of dynamic regimes and the other two $\gamma$ bifurcations have no effect on the number of dynamic regimes. The $\gamma$ bifurcations occurring at $D=0.0195$ and $D=0.0325$ only cause structural changes in the PDFs (Fig. 7a) to affect the mean passage time measure $\mathscr{R}_{i}^{\tau}$ (Fig. 7c).

Increasing $D$ has the general effect of increasing the regime size measure $\mathscr{R}_{i}^{r}$ for all dynamic regimes (Fig. 7c), with due accounting for the destruction and creation of dynamic regimes due to noise induced bifurcations. This is attributed to the fact that, a higher driver variability pushes the system farther, thereby enabling it to increase its regime size. The creation and destruction of dynamic regimes due to noise induced bifurcations causes the regime probability measures $\mathscr{R}_{i}^{R}$ to jump at bifurcation points. For example, the $\Delta$ bifurcation at $D=0.545$ destroys dynamic regimes 3 and 4 and transfers their probabilities to the remaining dynamic regimes 1 and 2 causing a jump in their regime probabilities. With respect to the regime probability measures $\mathscr{R}_{i}^{R}$, for the given set of parameters, we find that for driver variabilities beyond the $\Delta$ bifurcations, dynamic regimes with lower stable states are preferred, while for driver variability within the $\Delta$ bifurcations all dynamic regimes are almost equally likely. Unlike for the case of Gaussian white noise variability where the two regime probabilities converge to 0.5 at very large noise variability $\left(D>D_{t}\right)$, we cannot expect such a convergence for the case of dichotomous noise variability if asymmetry exists between the two $\gamma$ parameters.

In Example B, when we start with a vegetated cover increasing extremes in mortality and growth (stress and non-stress) favors dynamic regimes with higher (vegetated) fraction cover in terms of their regime probabilities until a threshold driver variability is crossed $(D=0.0545)$. When the stresses are higher than this bifurcation threshold, the system favors the desert dynamic regime with low vegetation cover. Increased variability in mortality and growth are expected due to climate change, where we could have: i) increased seedling mortality rates [22], ii) altered herbivory patterns [20], iii) temperature stresses, 
hail and flood damage [32] etc. Simultaneously, we can also experience increased growth rates due to elevated $\mathrm{CO}_{2}$ [47]. Hence, an understanding of this bifurcation threshold and its relative location to the present and future variability in climate forcings is critical in predicting the behavior of the state of ecosystems in future. Through the insights gained from this dynamical system example, we can identify ecosystems that are near the vicinity of this bifurcation threshold and prioritize our remediation and mitigation efforts accordingly.

Similar to the regime size measure, the stochastic ecological resilience measure $\mathscr{R}_{i}^{s E}$ increases with increasing $D$ values for all dynamic regimes (after accounting for bifurcations). This is attributed to the increase in regime size with increasing driver variability. The mean passage time measure $\mathscr{R}_{i}^{\tau}$ exhibits both increasing and decreasing trends with increasing $D$ values and is the only measure that is sensitive to all bifurcations.

As mentioned earlier, Markovian dichotomous noise allows us to change the frequency, intensity, variability and asymmetric bias of the driver. The changes in intensity aspect of driver have been investigated earlier (Fig. 7 a, b and c). The effect of changes in the frequency of driver variability is illustrated in Fig. $7 \mathrm{~d}$, e and $\mathrm{f}$. This is performed by changing the $\gamma$ parameters. Comparing Fig. $7 \mathrm{a}, \mathrm{b}$ and $\mathrm{c}$ with $\mathrm{d}$, e and $\mathrm{f}$, in the context of the example problems $\mathrm{A}$ and $\mathrm{B}$, we see that decreasing the frequency of driver variability by an order of magnitude results in increased regime probability for the vegetated dynamic regime and decreased regime probability for the intermediate vegetated regime (Fig. 7f, $\mathscr{R}_{i}^{R}$ ). This illustrates the effect of frequency on the stabilization or destabilization of vegetated regime. Furthermore, there is an invariant effect for the regime size resilience measure (Fig. $7 f, \mathscr{R}_{i}^{r}$ ) and an overall decrease in mean passage time resilience measure (Fig. $7 f, \mathscr{R}_{i}^{\tau}$ ). It can be observed that the $\gamma$ parameters exert a strong influence on the mean passage time. When we decrease $\gamma$ values, we decrease the switching frequency between the two dichotomous levels and increase the recovery time to each of the system states causing a decrease in the mean passage time. Hence, higher $\gamma$ values result in higher $\mathscr{R}_{i}^{\tau}$. This example illustrates converging, invariant, and diverging 
responses to changes in frequency of driver variability.

Figs.9 and 10 illustrate the effects of changing parameters and driver variability on resilience measures $\mathscr{R}_{i}^{r}, \mathscr{R}_{i}^{R}, \mathscr{R}_{i}^{s E}$ and $\mathscr{R}_{i}^{\tau}$ indicating the presence of noise induced bifurcations resulting in the creation and destruction of dynamic regimes. It can be observed from both the figures that the maximum variations in the resilience measures are observed near the regions where there are noise induced bifurcations (around $\theta_{1}=0$ and when $\theta_{2}<1.0$ ). Furthermore, the effect of the parameter boundary where catastrophic shift occurs in the deterministic conditions $\left(27 \theta_{1}^{2} \theta_{3}<4 \theta_{2}^{3}\right)$ is also strongly manifested in the resilience values.

The regime size measure $\mathscr{R}_{i}^{r}$ generally increases with increasing $D$ values and decreases with increasing $\left|\theta_{1}\right|$ values (Fig. 9a). A higher $D$ value enables the system to explore a larger domain thus increasing the regime size. Since bifurcations appear near $\theta_{1}=0$, we observe maximum regime size values around this region. The behavior of $\mathscr{R}_{i}^{r}$ (Fig. 9a) closely mimics the stochastic ecological resilience $\mathscr{R}_{i}^{s E}$ (Fig. 10a) for identical reasons.

The regime probability measure $\mathscr{R}_{i}^{R}$ shows an interesting variation with increasing $D$ values where, there is a general enhancement in dynamic regime preference as the driver variability increases (Fig. 9b). Dynamic regimes which are dominant at low $D$ values are more dominant at higher $D$ values, while dynamic regimes which are not dominant at low $D$ values become less dominant at higher $D$ values. This behavior is the opposite of the one we observe for the case of Gaussian white noise where we have a variability induced stabilization of weak dynamic regimes and destabilization of strong dynamic regimes. This illustrates the important role that the type of driver noise, its magnitude and variability play in determining the dynamical outcome of ecological systems.

While the mean passage time measure $\mathscr{R}_{i}^{\tau}$ shows variations similar to those of the other measures, with strong influence of the deterministic catastrophic shift condition and bifurcation condition (Fig. 10b), its behavior is more influenced by the values of $\gamma$ as discussed earlier. Furthermore, an asymmetry between $\gamma_{1}$ and $\gamma_{2}$ values would demonstrate a preference to the $\mathscr{R}_{i}^{\tau}$ measures. For our example in Fig. 10b, $\gamma_{1}>\gamma_{2}$ and hence transitions from $\Delta_{1}$ to $\Delta_{2}$ take shorter 
time than transitions from $\Delta_{2}$ to $\Delta_{1}$. These can be easily observed in regions where only 2 dynamic regimes are present, where $\mathscr{R}_{1}^{\tau}<\mathscr{R}_{2}^{\tau}$, and in regions where 4 dynamic regimes are present, where $\mathscr{R}_{3}^{\tau}<\mathscr{R}_{4}^{\tau}$. This is because $\mathscr{R}_{1}^{\tau}$ and $\mathscr{R}_{3}^{\tau}$ correspond to $\Delta_{1}$ levels and $\mathscr{R}_{2}^{\tau}$ and $\mathscr{R}_{4}^{\tau}$ correspond to the $\Delta_{2}$ levels. Interpreting this result in the context of Example B, we infer that, the mean switching frequency of driver variability from stress to non-stress conditions is higher than from non-stress to stress conditions. Hence, it takes a longer time to transition out of the vegetated cover than the desert cover.

Based on these analyses we infer that there are several attributes to characterize the resilience of a system and the various resilience measures capture independent and complementary aspects of the system dynamics. The interactions between the system dynamics and the driver characteristics creates emergent behavior that are different for different resilience measures. This makes it possible to arrive at the same resilience values through several different combinations of system and driver parameters. This is captured through the concept of iso-resilience described in the next section.

\section{Iso-resilience}

Iso-resilience curves are contour lines that connect regions in the parameter space that have identical resilience measure values. The parameter space can be generated from a selection of the system and driver parameters. Fig. 11 presents iso-resilience curves for measures $\mathscr{R}_{1}^{R}$ and $\mathscr{R}_{1}^{\tau}$ as a function of driver variability $D$ for a system driven by Gaussian white noise. Fig. 11a illustrates the strong non-linearities in the behavior of $\mathscr{R}_{1}^{R}$ as a function of $D$ and $\theta_{2}$. An interesting behavior can be observed where for a fixed $D, \mathscr{R}_{1}^{R}$ decreases, reaches a minima and then increases with increasing $\theta_{2}$ values. Applying this behavior to Example A, we find that under low emigration rates, the average fraction of time the system state lies in the refuge dynamic regime $\left(\mathscr{R}_{1}^{R}\right)$ initially decreases, but later increases with increasing population growth rates. Therefore, to maintain a constant $\mathscr{R}_{1}^{R}$ under increasing population growth rates, using the iso-resilience 
curves in Fig. 11a we can decipher that we have to decrease $D$ when $D \lesssim$ 0.03 but increase $D$ when $D \gtrsim 0.03$. $\mathscr{R}_{1}^{R}$ exhibits a monotonously decreasing behavior when the parameter $\theta_{1}$ is increased (Fig. 11c) whereas $\mathscr{R}_{1}^{\tau}$ exhibits a different monotonously increasing behavior when the parameter $\theta_{2}$ is increased (Fig. 11d). This further illustrates that changing parameters can increase one attribute of resilience while decreasing another attribute.

Iso-resilience curves can also be developed for derived resilience measures such as regime dominance, obtained as the difference in regime probabilities between two dynamic regimes, for example $\mathscr{R}_{1}^{R}-\mathscr{R}_{2}^{R}$ (Fig. 11b). Regime dominance measures the average 'excess' fraction of time that the system state stays in one dynamic regime compared to another dynamic regime. A high value of regime dominance can indicate that the system state shows strong preference towards one dynamic regime over another.

\section{Discussion}

In this section, we highlight some potential applications of our resilience characterization in other catastrophic shift systems observed in nature. An example of two different driver variables, fire and grazing on grasslands is discussed by Anderies et al. [1]. Depending on the particular set of parameters chosen, Anderies et al. [1] show that fire causes a stabilization effect, while grazing causes a destabilization effect on the grassland dynamic regime. The regime probability measure $\mathscr{R}_{i}^{R}$ captures this effect and the concept of variability induced stabilization, destabilization, or enhancement of dynamic regimes can be inferred in the example of Anderies et al. [1]. In this context, we would like to point out that the popular notion 'by controlling variability we shrink ecological resilience' [18] is only partially true. As demonstrated from our simulations, depending on the system dynamics (parameters), the type and intensity of driver variation, and the particular attribute of resilience, we can have either an increasing, decreasing, or invariant resilience behavior to increasing driver variability for each and every dynamic regime. 
Oftentimes, there are conflicts between short term gains and long term resilience in resource exploitation, as in the case of using excessive fertilizer and pesticides, plowing, overstocking etc. in agricultural practices [7]. Similar resilience versus cost trade offs in other fields such as component failure of complex machines, resistance to diseases, resilience specialization to one type of driver variability versus another etc. have also been highlighted [28]. An interesting case of such a trade off in conservation versus resilience in the case of marine coral ecosystems is discussed in Cote and Darling [8]. In their paper Cote and Darling [8] argue that, the development of marine conservation sanctuaries for the protection of coral ecosystems from increased variability in environmental forcings such as temperature changes, nutrient loading etc. could potentially result in decreasing the resilience of the ecosystem to extreme events. This is because, a coral ecosystem which was not protected from environmental variability, although will be in a slightly degraded state, has a higher resilience to extreme events. While Cote and Darling [8] claim that such phenomenon are plausible, we suggest that using the measures developed in this work, we can determine parameter conditions where this behavior is expected to occur, and whether increased driver variability stabilizes or destabilizes the current dynamic regime.

The framework developed in this paper enables us to gain insights regarding several important questions in the context of catastrophic shift systems. For example, in shallow lake ecosystems, it is important to know whether interspecies competition or intrinsic growth rate dominates the system dynamics in the occurrence of algal blooms [3]. These two processes can be captured by our parameters $\theta_{2}$ and $\theta_{3}$ and using plots similar to Fig. 3 and Fig. 11, one can identify regions in the resilience landscape that indicate dominance of different processes. Such characterizations can act as powerful tools to manage lake ecosystems from catastrophic transitions.

An interesting application of iso-resilience curves is that it enables us to devise paths for traversing the resilience landscape under changing parameter conditions. Specific paths can be designed that incorporate constraints such as, 
avoiding unfavorable regions and passing through favored regions [16, 17, 19]. For example, in Fig. 11a, if we start at Z1 and the $D$ is increased from 0.02 to 0.08 , in order to maintain the same regime probability $\mathscr{R}_{1}^{R}=0.98, \theta_{2}$ has to be decreased to 0.3 (represented as Z2). One possible path to accomplish this transition is indicated by the straight arrow. However, if we impose an additional constraint that $\mathscr{R}_{1}^{R}>0.8$ during the entire course of this transition, then we have to follow the path indicated by the bent arrow. The critical condition that must be met in this case is that $\theta_{2}<0.3$ before $D>0.044$. Iso-resilience curves enable us to identify such critical points or bottlenecks when designing travel paths in a resilience landscape and hence can be used as valuable tools in managing resilience aspects of non-linear dynamical systems $[16,25,18]$.

\section{Conclusions}

In this study, we use the canonical catastrophic shift system to develop a system-driver-attribute triplet framework to quantify resilience. This framework captures the interplay between intrinsic system dynamics and extrinsic drivers. The concept of resilience does not just focus on the state of the system, but more importantly, the process dynamics and feedbacks which are captured by the DOA or dynamic regime. We propose several different resilience measures and interpret their variation to changing parameters through the help of two ecological examples; (i) bistable population dynamics (Example A) (ii) multi-stable vegetation cover dynamics (Example B). Furthermore, we develop iso-resilience curves and use them to design travel paths in a resilience landscape, providing powerful tools to manage DOA or dynamic regime transitions in catastrophic shift systems. Several interesting behaviors were also observed through the help of these simulations. These include:

- Variability induced stabilization of weak dynamic regimes

- Variability induced enhancement of dynamic regime dominance 
- Regions of sensitivity

- Barrier regions

- Trap or escape regions

- Bifurcation thresholds

- Bottlenecks in travel paths of resilience landscapes

In this work we have presented resilience measures for a univariate dynamical system corresponding to a canonical catastrophic shift system, at aggregate spatial scales and stationary time scales. Measures are developed for two cases: when the system is subject to instantaneous shock in drivers which are non interacting (the system relaxation time is shorter than the inter-arrival times of the shocks), and continuous varying drivers where the characteristics of driver variability gets inter-twined with the system dynamics. The mathematical framework presented in this study lends itself for extension to a multivariate dynamical system by making $x$ a vector of variables. Similarly, by introducing spatial derivatives in equation (10) we can enable the model to capture aspects of spatial heterogeneity in the resilience of the dynamical system. Furthermore, analyzing the temporal evolution of the PDF would enable us to analyze the time dependent nature of resilience under a stochastic noise. More importantly this will also provide insights into the dependence on initial conditions to resilience measures.

While we only analyze catastrophic shift systems in this paper, the mathematical framework presented can be readily applied to other non-linear dynamical systems including multi-state systems subject to multiplicative stochastic noise terms. It must be noted that, in the examples presented in the present study, only additive stochastic noise in system drivers are considered. Including the effects of multiplicative noise, enhances the complexity of the dynamical system (stochastic resonance, coherence, ratchet effect, etc.) and its associated DOA or dynamic regimes leading to the emergence of other interesting behaviors such as oscillations, pattern formations etc. [4, 39]. Furthermore, extending to 
multiple dimensions would enable us to explore the quantification of more challenging resilience aspects such as adaptability, ability to self organize, learning, etc.

\section{Acknowledgments}

This work was supported by NSF grants ATM 06-28687, EAR 09-11205, and CBET 12-0942.

\section{References}

[1] Anderies, J.M., Janssen, M.A., Walker, B., 2002. Grazing management, resilience and the dynamics of fire driven rangeland system. Ecosystems 5, $23-44$.

[2] Anderies, J.M., Walker, B., Kinzig, A., 2006. Fifteen weddings and a funeral: case studies and resilience based management. Ecology and Society $11,21$.

[3] Angeler, D.G., Trigal, C., Drakare, S., Johnson, R.K., Goedkoop, W., 2010. Identifying resilience mechanisms to recurrent ecosystem perturbations. Oceologia 164, 231-241.

[4] Anishchenko, V.S., Astakhov, V., Neiman, A., Vadivasova, N., SchimanskyGeier, L., 2002. Nonlinear dynamics of chaotic and stochastic systems, tutorial and modern developments. Springer-Verlag Press, Berlin Heidelberg, Germany.

[5] Bellwood, D.R., Huges, T.P., Folke, C., Nystro, M., 2004. Confronting the coral reef crisis. Nature 429, 827-833.

[6] Brand, F.S., Jax, L., 2007. Focusing the meaning(s) of resilience: resilience as a descriptive concept and a boundary object. Ecology and Society . 
[7] Carpenter, S.R., Walker, B., Anderies, J.M., Abel, N., 2001. From metaphor to measurement: Resilience of what to what? Ecosystems 4, $765-781$.

[8] Cote, I.M., Darling, E.S., 2010. Rethinking ecosystem resilience in the face of climate change. PLOS Biology 8.

[9] Cumming, G.S., Barnes, G., Perz, S., Schmink, M., Seiving, E., Southworth, J., Binford, M., Holt, R.D., Stickler, C., VanHolt, T., 2005. An exploratory framework for the empirical measurement of resilience. Ecosystems 8, 975-987.

[10] Dent, C.L., Graeme, S., R.Carpenter, S., 2002. Multiple states in river and lake ecosystems. Philosophical Transactions of the Royal Society 357, 635-645.

[11] D'Odorico, P., Bhattachan, A., 2012. Hydrologic variability in dryland regions: impacts on ecosystem dynamics and food security. Philosophical Transactions of the Royal Society B-Biological Sciences 367, 3145-3157.

[12] Fath, B.D., Cabezas, H., Pawlowski, C.W., 2003. Regime changes in ecological systems: an information theory approach. Journal of Theoretical Biology 222, 517-530.

[13] Fath, B.D., Cabezas, H., Pawlowski, C.W., 2006. Regime changes in ecological systems: an information theory approach. Ecological Modelling 195, $72-82$.

[14] Field, C.B., Barros, V., Stocker, T.F., Qin, D., Dokken, D.J., Ebi, K.L., Mastrandrea, M.D., Mach, K.J., Plattner, G.K., Allen, S.K., Tignor, M., Midgley, P.M., 2012. Managing the Risks of Extreme Events and Disasters to Advance Climate Change Adaptation. Technical Report. Intergovernmental Pannel on Climate Change. New York, NY.

[15] Folke, C., 2006. Resilience: the emergence of a perspective for socialecological systems analyses. Global Environmental Change 16, 253-267. 
[16] Folke, C., Carpenter, S.R., Walker, B., Scheffer, M., Elmqvist, T., Gunderson, L., Holling, C.S., 2004. Regime shifts, resilience and biodiversity in ecosystem management. Annual Review of Ecology, Evolution, and Systematics $35,557-581$.

[17] Folke, C., Others, 2002. Resilience and Sustainable Development: building adaptive capacity in a world of transformations. Technical Report. Environmental Advisory Council.

[18] Gunderson, L.H., 2009. Ecological resilience in theory and application. Annual Review of Ecology, Evolution, and Systematics 31, 425-439.

[19] Gunderson, L.H., Holling, C.S., 2002. Panarchy: Understanding Transformations in Human and Natural Systems. Island Press, Washington, DC.

[20] Hamilton, J.G., Dermody, O., Aledea, M., amd A. Rogers amd M. R. Berenbaum, A.R.Z., Delucia, E.H., 2005. Anthropogenic changes in tropospheric composition increase susceptibility of soybean to insect herbivory. Environmental Entomology 34, 479-485.

[21] Higgins, S.I., Bond, W.J., Trollope, W., 2000. Fire, resprouting and variability: a recipe for grass-tree coexistence in savanna. Journal of Ecology $88,213-229$.

[22] Hirota, M., Holmgren, M., van Nes, E.H., Scheffer, M., 2012. Global resilience of tropical forest and savanna to critical transitions. Science 334, 232-235.

[23] Holling, C.S., 1973. Resilience and stability of ecological systems. Annual Review of Ecology and Systematics 4, 1-23.

[24] Holling, C.S., 1996. Engineering resilience versus ecological resilience, in: Schulze, P. (Ed.), Engineering within ecological constraints. National academies press, Washington, DC. chapter 2, pp. 31-44. 
[25] Holmgren, M., Scheffer, M., 2001. El nino as a window of opportunity for the restoration of degraded arid ecosystems. Ecosystems 4, 151-159.

[26] Horsthemke, W., Lefever, R., 2006. Noise induced transitions, theory and applications in physics, chemistry and biology. Springer-Verlag Press, Berlin Heidelberg, Germany.

[27] Janssen, M.A., Schoonc, M.L., Kee, W., Borner, K., 2006. Scholarly networks on resilience, vulnerability and adaptation within the human dimensions of global environmental change. Global Environmental Change 16, 240-252.

[28] Kitano, H., 2004. Biological robustness. Nature Reviews Genetics 5, 826837.

[29] Knapp, A.K., Fay, P.A., Blair, J.M., Collins, S.L., Smith, M.D., Carlisle, J.D., Harper, C.W., Danner, B.T., Lett, M.S., McCarron, J.K., 2002. Rainfall variability, carbon cycling, and plant species diversity in a mesic grassland. Science 298, 2202-2205.

[30] Kumar, P., 2001. Typology of hydrologic predictability. Water Resources Research 47.

[31] Kumar, P., 2013. Seasonal rain changes. Nature Climate Change 3, 783784.

[32] Mahoney, K., Alexander, M.A., Thompson, G., Barsugli, G.J., Scott, J.D., 2012. Changes in hail and flood risk in high-resolution simulations over colorados mountains. Nature Climate Change 2, 125-131.

[33] Marani, M., Lio, C.D., D'Alpaos, A., 2013. Vegetation engineers marsh morphology through multiple competing stable states. Proceedings National Academy Science USA 110, 3259-3263.

[34] May, R.M., 1973. Stability and complexity in model ecosystems. Princeton University Press, Princeton, NJ. 
[35] Morin, P.J., 1999. Community ecology. Blackwell Science Inc, Malden, MA.

[36] Pall, P., Aina, T., Stone, D.A., Stott, P.A., Nozawa, T., Hilberts, A.J., Lohmann, D., Allen, M.R., 2011. Anthropogenic greenhouse gas contribution to flood risk in england and wales in autumn 2000. Nature 470, 382-386.

[37] Pearl, R.L., Reed, L.J., 1920. On the rate of growth of the population of united states since 1790 and its mathematical representation. Proceedings National Academy Science USA 6, 275-288.

[38] Perrings, C., 2006. Resilience and sustainable development. Environment and Development Economics 11, 417-427.

[39] Ridolfi, L., D'odorico, P., Laio, F., 2011. Noise induced phenomena in the environmental sciences. Cambridge university Press, New York, NY.

[40] Rodriguez-Iturbe, I., Entekahabi, D., Bras, R.L., 1991. Nonlinear dynamics of soil moisture at climate scales 1 . stochastic analysis. Water Resources Research 27, 1899-1906.

[41] Sancho, J.M., 1985. External dichotomous noise: The problem of the mean first-passage time. Physical Review A 31, 3523-3525.

[42] Schar, C., Visale, P.L., Luthi, D., Frei, C., Haberli, C., Liniger, M.A., Appenzeller, C., 2004. The role of increasing temperature variability in european summer heatwaves. Nature 427, 332-336.

[43] Scheffer, M., Carpenter, S.R., 2003. Catastrophic regime shifts in ecosystems: linking theory to observation. TRENDS in Ecology and Evolution $18,648-656$.

[44] Scheffer, M., Carpenter, S.R., Foley, J.A., Folke, C., Walker, B., 2001. Catastrophic shifts in ecosystems. Nature 413, 591-596. 
[45] Scheffer, M., Hirota, M., Holmgren, M., Nes, E.H.V., Chapin, F.S., 2012. Thresholds for boreal biome transitions. Proceedings National Academy Science USA 109, 21384-21389.

[46] Schoener, T.W., 1983. Field experiments on interspecific competition. American naturalizst 122, 240-285.

[47] Solomon, S., Qin, D., Manning, M., Chen, Z., Marquis, M., Averyt, K.B., Tignor, M., Miller, H.L., 2007. Contribution of Working Group I to the Fourth Assessment Report of the Intergovernmental Panel on Climate Change. Technical Report. Intergovernmental Pannel on Climate Change. New York, NY.

[48] Steffen, W.S., Sanderson, A., Tyson, P.D., Jager, J., Matson, P.A., Moore, B., Oldfield, F., Richardson, K., Schellnhuber, H.J., Turner, B.L., Wasson, R.J., 2004. Global change and the earth system: A planet under pressure. Springer-Verlag Press, Berlin Heidelberg, Germany.

[49] Stott, P.A., Stone, D.A., Allen, M.P., 2004. Human contribution to the european heatwave of 2003 . Nature 427, 610-614.

[50] Strogatz, S.H., 1994. Nonlinear dynamics and chaos with applications to physics, biology, chemistry and engineering. Westview Press, Cambridge, MA.

[51] Tilman, D., Downing, J.A., 1994. Biodiversity and stability in grasslands. Nature 367, 363-365.

[52] Timmermann, A., Oberhuber, J., Bacher, A., Esch, M., Latif, M., Roeckner, E., 2011. Increased el nino frequency in a climate model forced by future greenhouse warming. Nature 398, 694-697.

[53] Turner, B.L., 2010. Vulnerability and resilience: Coalescing or paralleling approaches for sustainability science? Global Environmental Change 20, $570-576$. 
[54] Turner, B.L., Kasperson, R.E., Matsone, P.A., McCarthy, J.J., Corell, R.W., Christensen, L., Eckley, N., Kasperson, J.X., Luers, A., ML, M.L.M., Polsky, C., Pulsipher, A., Schiller, A., 2006. A framework for vulnerability analysis in sustainability science. Proceedings National Academy Science USA $100,8074-8079$.

[55] Walker, B., C. S. Folling, C., Carpenter, S.R., Kinzig, A., 2004. Resilience, adaptability and transformability in social-ecological systems. Ecology and Society . 


\begin{tabular}{|c|c|c|c|c|}
\hline Driver & $\begin{array}{l}\text { Name of } \\
\text { measure }\end{array}$ & Variable & Definition & Explanation \\
\hline \multirow{5}{*}{ 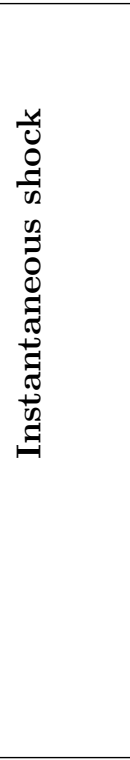 } & Size of DOA & $\mathscr{R}_{i}^{s}$ & $\left|\stackrel{\circ}{x}_{i, j}-\stackrel{\circ}{x}_{i, k}\right|[Q]$ & $\begin{array}{l}\text { Size of the domain of attraction of } X_{i} \\
\text { where the attractor is } \dot{x}_{i} \text {. }\end{array}$ \\
\hline & $\begin{array}{l}\text { Ecological re- } \\
\text { silience }\end{array}$ & $\mathscr{R}_{i}^{E}$ & $\min _{j}\left\{\left|\stackrel{\bullet}{x}_{i}-\stackrel{\circ}{x}_{i, j}\right|\right\} \quad[Q]$ & $\begin{array}{l}\text { Minimum distance between the at- } \\
\text { tractor } \dot{x}_{i} \text { and the unstable boundary } \\
\stackrel{\circ}{x}_{i, j} \text {. }\end{array}$ \\
\hline & $\begin{array}{l}\text { Engineering re- } \\
\text { silience }\end{array}$ & $\mathscr{R}_{i}^{e}$ & $\min _{j}\left\{\frac{1}{T_{i, j}}\right\} \quad[1 / T]$ & $\begin{array}{l}\text { Minimum of the inverse of the time } \\
\text { taken for the system state to travel } \\
\text { from the unstable boundary } \stackrel{\circ}{x}_{i, j} \text { to } \\
\text { the attractor } \dot{x}_{i} \text { subject to a } \epsilon \text { per- } \\
\text { turbation. }\end{array}$ \\
\hline & $\begin{array}{l}\text { Attractor } \\
\text { response rate }\end{array}$ & $\mathscr{R}_{i}^{\lambda}$ & $\left|\frac{d F(x)}{d x}\right|_{x=\dot{x}_{i}}[1 / T]$ & $\begin{array}{l}\text { Rate at which the system state in } \\
\text { DOA } X_{i} \text { returns to its attractor } \dot{x}_{i} \\
\text { when subject to small perturbations. }\end{array}$ \\
\hline & $\begin{array}{l}\text { Activation po- } \\
\text { tential }\end{array}$ & $\mathscr{R}_{i}^{a}$ & $\begin{array}{l}\min _{j}\left\{V\left(\stackrel{\circ}{x}_{i, j}\right)-V\left(\dot{x}_{i}\right)\right\} \\
{\left[Q^{2} / T\right]}\end{array}$ & $\begin{array}{l}\text { Minimum potential required (using } \\
\text { potential function) to excite the sys- } \\
\text { tem from an attractor } \dot{x}_{i} \text { to the un- } \\
\text { stable boundary } \stackrel{\circ}{x}_{i, j} \text {. }\end{array}$ \\
\hline \multirow{4}{*}{ 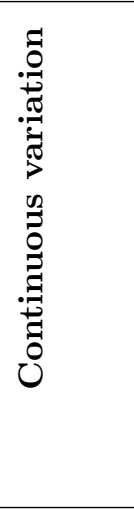 } & Regime size & $\mathscr{R}_{i}^{r}$ & $\left|\stackrel{\circ}{x}_{i, j}-\stackrel{\circ}{x}_{i, k}\right|[Q]$ & $\begin{array}{l}\text { Size of the regime } X_{i} \text { where the mode } \\
\text { is } \dot{x}_{i} \text {. }\end{array}$ \\
\hline & $\begin{array}{l}\text { Regime proba- } \\
\text { bility }\end{array}$ & $\mathscr{R}_{i}^{R}$ & $\int_{X_{i}} P_{S}(x) d x \quad[-]$ & $\begin{array}{l}\text { Probability of system state in regime } \\
X_{i} \text {. For connected regimes, this also } \\
\text { represents the average fraction of time } \\
\text { spent in regime } X_{i} \text {. }\end{array}$ \\
\hline & $\begin{array}{l}\text { Stochastic } \\
\text { ecological } \\
\text { resilience }\end{array}$ & $\mathscr{R}_{i}^{s E}$ & $\min _{j}\left\{\left|\dot{x}_{i}-\stackrel{\circ}{x}_{i, j}\right|\right\} \quad[Q]$ & $\begin{array}{l}\text { Minimum distance between the mode } \\
\dot{x}_{i} \text { and the regime boundary } \stackrel{\leftrightarrow}{x}_{i, j} \text {. }\end{array}$ \\
\hline & $\begin{array}{l}\text { Mean passage } \\
\text { time }\end{array}$ & $\mathscr{R}_{i}^{\tau}$ & $\min _{j}\left\{\tau_{i, j}\right\} \quad[T]$ & $\begin{array}{l}\text { Average time spent before a system } \\
\text { state in regime } X_{i} \text { transitions to tran- } \\
\text { sition to regime } X_{j} \text {. }\end{array}$ \\
\hline
\end{tabular}

Table 1: List of resilience measure formulations 

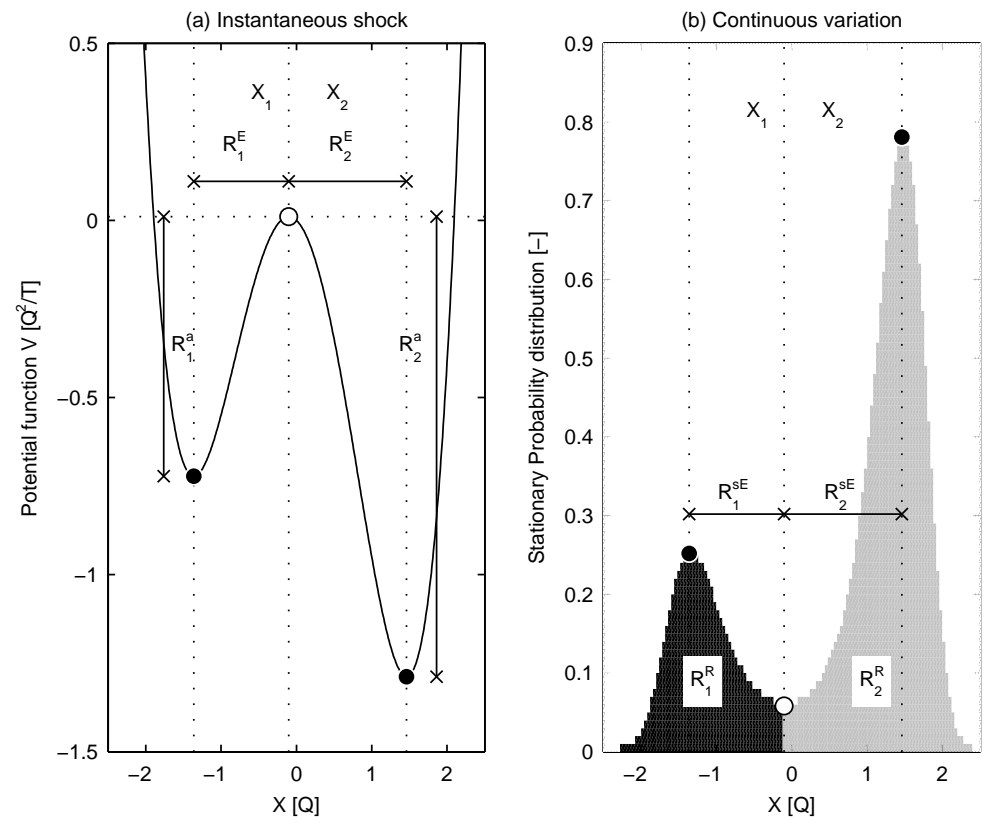

Figure 1: Concepts of (a) DOA for instantaneous shock (equation (7)) and (b) dynamic regime for continuous variability is system drivers (equation (10)) for a catastrophic shift system. The system parameters used for both (a) and (b) are $\theta_{1}=0.2, \theta_{2}=2.0$ and $\theta_{3}=-1.0$ and the additional parameter for (b) is Gaussian white noise scale $D=0.5$. When the system is subject to an instantaneous shock in the driver, DOAs can be obtained from a potential function $V(x)$ plot such as in (a) where the peaks in the potential function (occurring at the unstable fixed point $\stackrel{\circ}{x}_{1,2}=-0.10$ ) represented by the white circle separate the two DOAs. The troughs of the potential function (occurring at the stable fixed points $\dot{x}_{1}=-1.36$ and $\dot{x}_{2}=1.46$ ) are represented by the black circles. The ecological resilience $\mathscr{R}_{1}^{E}(1.26)$ and $\mathscr{R}_{2}^{E}(1.56)$ and the activation potential $\mathscr{R}_{1}^{a}(0.73)$ and $\mathscr{R}_{2}^{a}(1.30)$ resilience measures for the two DOAs are illustrated. When the system is subject to a continuous variability in the driver, dynamic regimes can be obtained from a stationary PDF such as in (b) where the troughs in the PDFs (occurring at the anti-mode $\stackrel{\circ}{x}_{1,2}=-0.10$ ) represented by the white circle separate the two dynamic regimes. The peaks of the PDF (occurring at the stable fixed points $\dot{x}_{1}=-1.36$ and $\dot{x}_{2}=1.46$ are represented by the black circles. The stochastic ecological resilience $\mathscr{R}_{1}^{s E}$ (1.26) and $\mathscr{R}_{2}^{s E}(1.56)$ and the regime probability $\mathscr{R}_{1}^{R}(0.27)$ and $\mathscr{R}_{1}^{a}(0.73)$ resilience measures for the two dynamic regimes are also illustrated. 


\begin{tabular}{|c|c|c|}
\hline Driver & $\begin{array}{l}\text { Illustration of } \\
\text { DOA or regime }\end{array}$ & $\begin{array}{l}\text { Measure } \\
\text { comparison }\end{array}$ \\
\hline \multirow{4}{*}{ 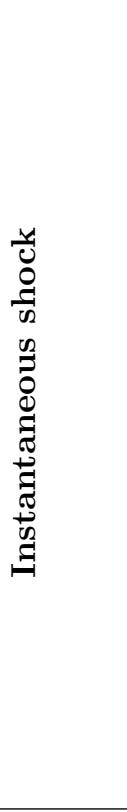 } & $\underset{\substack{\mathbb{O} \\
>}}{\stackrel{0}{>}}$ & $\begin{array}{l}\mathscr{R}_{1}^{s}=\mathscr{R}_{1^{\prime}}^{s} \\
\mathscr{R}_{1}^{E}>\mathscr{R}_{1^{\prime}}^{E} \\
\mathscr{R}_{1}^{a}>\mathscr{R}_{1^{\prime}}^{a}\end{array}$ \\
\hline & (b) & $\begin{array}{c}\mathscr{R}_{1}^{s}=\mathscr{R}_{1^{\prime}}^{s} \\
\mathscr{R}_{1}^{E}=\mathscr{R}_{1^{\prime}} \\
\mathscr{R}_{1}^{a}=\mathscr{R}_{1^{\prime}}^{a} \\
\mathscr{R}_{1}^{\lambda}<\mathscr{R}_{1^{\prime}}^{\lambda} \\
\mathscr{R}_{1}^{e}<\mathscr{R}_{1^{\prime}}^{e}\end{array}$ \\
\hline & 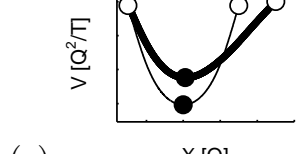 & $\begin{array}{l}\mathscr{R}_{1}^{s}<\mathscr{R}_{1^{\prime}}^{s} \\
\mathscr{R}_{1}^{E}=\mathscr{R}_{1^{\prime}}^{E} \\
\mathscr{R}_{1}^{a}=\mathscr{R}_{1^{\prime}}^{a}\end{array}$ \\
\hline & 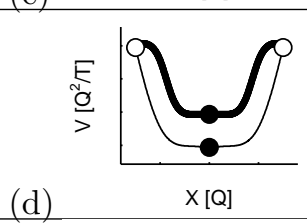 & $\begin{array}{c}\mathscr{R}_{1}^{s}=\mathscr{R}_{1^{\prime}}^{s} \\
\mathscr{R}_{1}^{E}=\mathscr{R}_{1^{\prime}}^{E} \\
\mathscr{R}_{1}^{a}>\mathscr{R}_{1^{\prime}}^{a} \\
\mathscr{R}_{1}^{\lambda}=\mathscr{R}_{1^{\prime}}^{\lambda} \\
\mathscr{R}_{1}^{e}>\mathscr{R}_{1^{\prime}}^{e}\end{array}$ \\
\hline \multirow{3}{*}{ 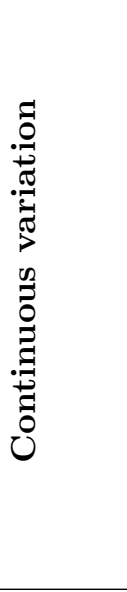 } & $\begin{array}{l}\frac{T}{u} \\
\frac{u}{a}\end{array}$ & $\begin{array}{l}\mathscr{R}_{1}^{r}=\mathscr{R}_{1^{\prime}}^{r} \\
\mathscr{R}_{1}^{R}>\mathscr{R}_{1^{\prime}}^{R} \\
\mathscr{R}_{1}^{s E}=\mathscr{R}_{1^{\prime}}^{s E}\end{array}$ \\
\hline & $(\mathrm{f})$ & $\begin{array}{l}\mathscr{R}_{1}^{r}=\mathscr{R}_{1^{\prime}}^{r} \\
\mathscr{R}_{1}^{R}=\mathscr{R}_{1^{\prime}}^{R} \\
\mathscr{R}_{1}^{s E}<\mathscr{R}_{1^{\prime}}^{s E}\end{array}$ \\
\hline & $(\mathrm{g})$ & $\begin{array}{l}\mathscr{R}_{1}^{r}>\mathscr{R}_{1^{\prime}}^{r} \\
\mathscr{R}_{1}^{R}<\mathscr{R}_{1^{\prime}}^{R} \\
\mathscr{R}_{1}^{s E}=\mathscr{R}_{1^{\prime}}^{s E}\end{array}$ \\
\hline
\end{tabular}

Figure 2: Conceptual illustration of the ability of the different resilience measures to capture various resilience attributes of a dynamical system when subjected to instantaneous shocks and continuous variation in system drivers. White circles represent DOA or dynamic regime boundaries, and black circles represent stable fixed points or modes of the corresponding DOA or dynamic regime. Thin and thick lines correspond to two alternate shapes of potential functions or PDFs, identified as 1 and $1^{\prime}$ respectively, used for comparison. 

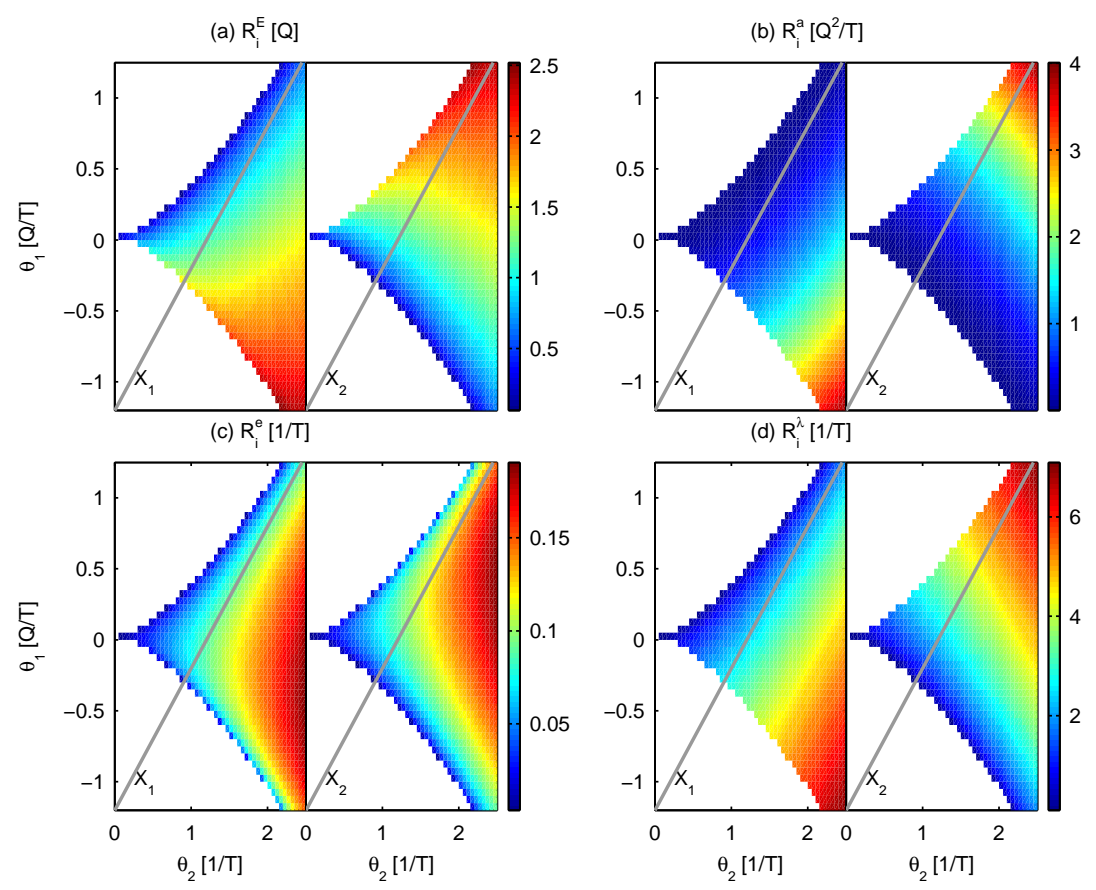

Figure 3: Variation of (a) Ecological resilience $\mathscr{R}_{i}^{E}[Q]$, (b) Activation potential $\mathscr{R}_{i}^{a}\left[Q^{2} / T\right]$, (c) Engineering resilience $\mathscr{R}_{i}^{e}[1 / T]$ and (d) Attractor response rate $\mathscr{R}_{i}^{\lambda}[1 / T]$ resilience measures for a catastrophic shift system as a function of imperfection parameter $\theta_{1}$ and rate parameter $\theta_{2}$ for the two DOAs $X_{1}$ and $X_{2}$ when subject to an instantaneous shock in system driver. DOAs $X_{1}$ and $X_{2}$ correspond to the lower and higher attractor value $\dot{x}_{1}$ and $\dot{x}_{2}$ respectively. White space represents parameter combinations for which the system does not exhibit catastrophic shift behavior $27 \theta_{1}^{2} \theta_{3}>4 \theta_{2}^{3}$. The diagonal grey line indicates slopes of constant immigration or emigration rates $I$ [number/year] in Example A problem or deforestation and reforestation rates $A\left[\mathrm{~m}^{2} /\right.$ year $]$ in Example B. 

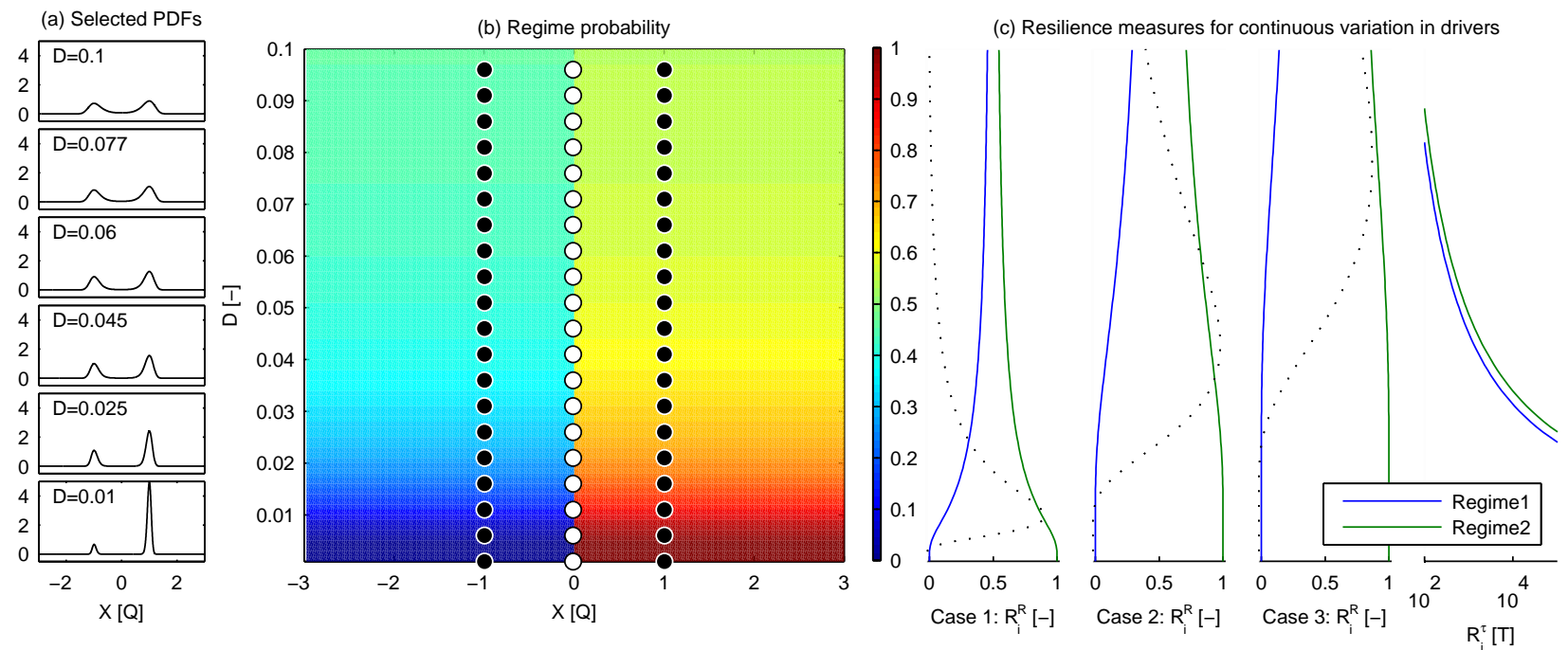

Figure 4: Effect of Gaussian white noise variability in the system driver on resilience measures at different intensities of driver variability on a catastrophic shift system with $\theta_{1}=0.01, \theta_{2}=1.0$ and $\theta_{3}=-1.0$ bounded by $\bar{x}_{1}=-\infty$ and $\bar{x}_{2}=\infty$. (a) Sample PDFs for different magnitudes of driver variability $D$ illustrate the effect of leveling of the PDFs as $D$ increases. (b) Regime probabilities $\mathscr{R}_{i}^{R}$ (color coded) as a function of driver variability $D$ along with the locations of modes $\dot{x}_{1}=-1.00$ and $\dot{x}_{2}=1.01$ (black circles) and anti-modes $\stackrel{\circ}{x}_{12}=-0.01$ (white circles). For all driver variability scales we have only two dynamic regimes $X_{1}$ and $X_{2}$. (c) Regime probability $\mathscr{R}_{i}^{R}$ for case $1: \theta_{1}=0.01$, for case $2: \theta_{1}=0.05$ and for case 3 : $\theta_{1}=0.1$ and mean passage time $\mathscr{R}_{i}^{\tau}$ resilience measures as a function of driver variability $D$. The dotted lines in the three $\mathscr{R}_{i}^{R}$ plots are $\left|\frac{d \mathscr{R}_{i}^{R}}{d D}\right|$ values (not to scale) representing the sensitivity of $\mathscr{R}_{i}^{R}$ to changing $D$ values. Note the effect of driver induced enhancement of regime probabilities of the weaker dynamic regime $X_{1}$ at the cost of the stronger dynamic regime $X_{2}$ in all three $\mathscr{R}_{i}^{R}$ plots in (c). Comparison between the three $\mathscr{R}_{i}^{R}$ plots demonstrates differences in the sensitivity regions of $\mathscr{R}_{i}^{R}$ to changing $D$ values. The peak of the $\left|\frac{d \mathscr{R}_{i}^{R}}{d D}\right|$ (dotted lines in (c)) represent regions of maximum sensitivity. The regime size measure $\mathscr{R}_{i}^{s}$ (not shown in figure) is $\infty$ for both dynamic regimes since the dynamic regimes are semi-infinite. The stochastic ecological resilience measures $\mathscr{R}_{1}^{s E}$ and $\mathscr{R}_{2}^{s E}$ (not shown in figure) have a constant value at 0.985 and 1.015 respectively for all $D$ values. Note that for illustration purposes, the upper and lower bounds for the $\mathscr{R}_{i}^{\tau}$ plot in (c) have been cropped. 

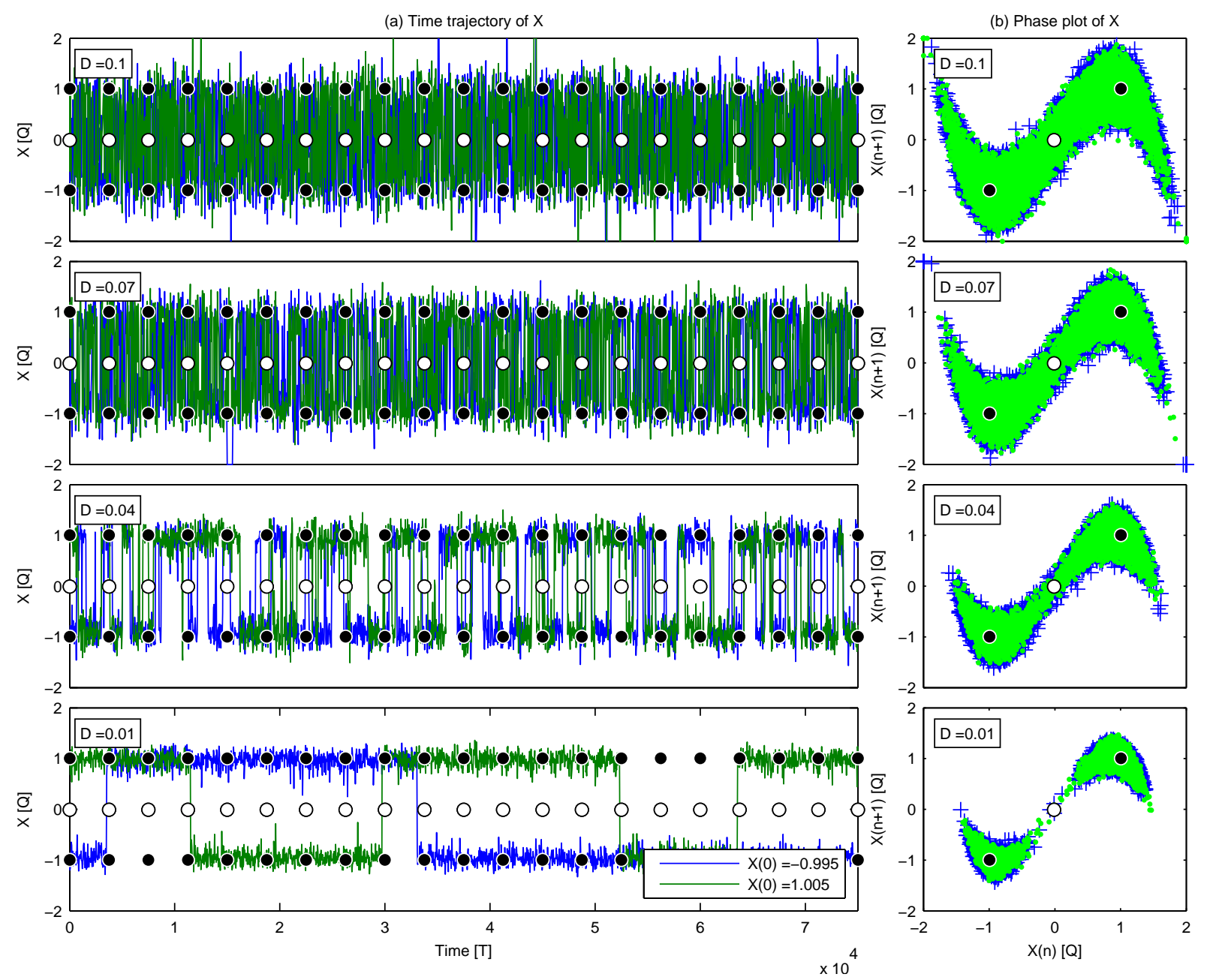

Figure 5: Effect of Gaussian white noise variability in the system driver on the time trajectory of the system state $x$ at different intensities of driver variability on a catastrophic shift system with $\theta_{1}=0.01, \theta_{2}=1.0$ and $\theta_{3}=-1.0$ bounded by $\bar{x}_{1}=-\infty$ and $\bar{x}_{2}=\infty$. Two different initial conditions at time $t=0$ are selected; the blue line corresponds to $x(0)=-0.995$ and the green line corresponds to $x(0)=1.005$. (a) Time trajectories for different magnitudes of driver variability $D$ illustrate the effect of increased switching between dynamic regimes and a corresponding decrease in $\mathscr{R}_{i}^{\tau}$ as $D$ increases. (b) Phase plots illustrating the range of values that the system state can take when subjected to Gaussian white noise. The locations of the modes of the PDF are represented as black circles and the locations of the anti-modes are represented as white circles. 

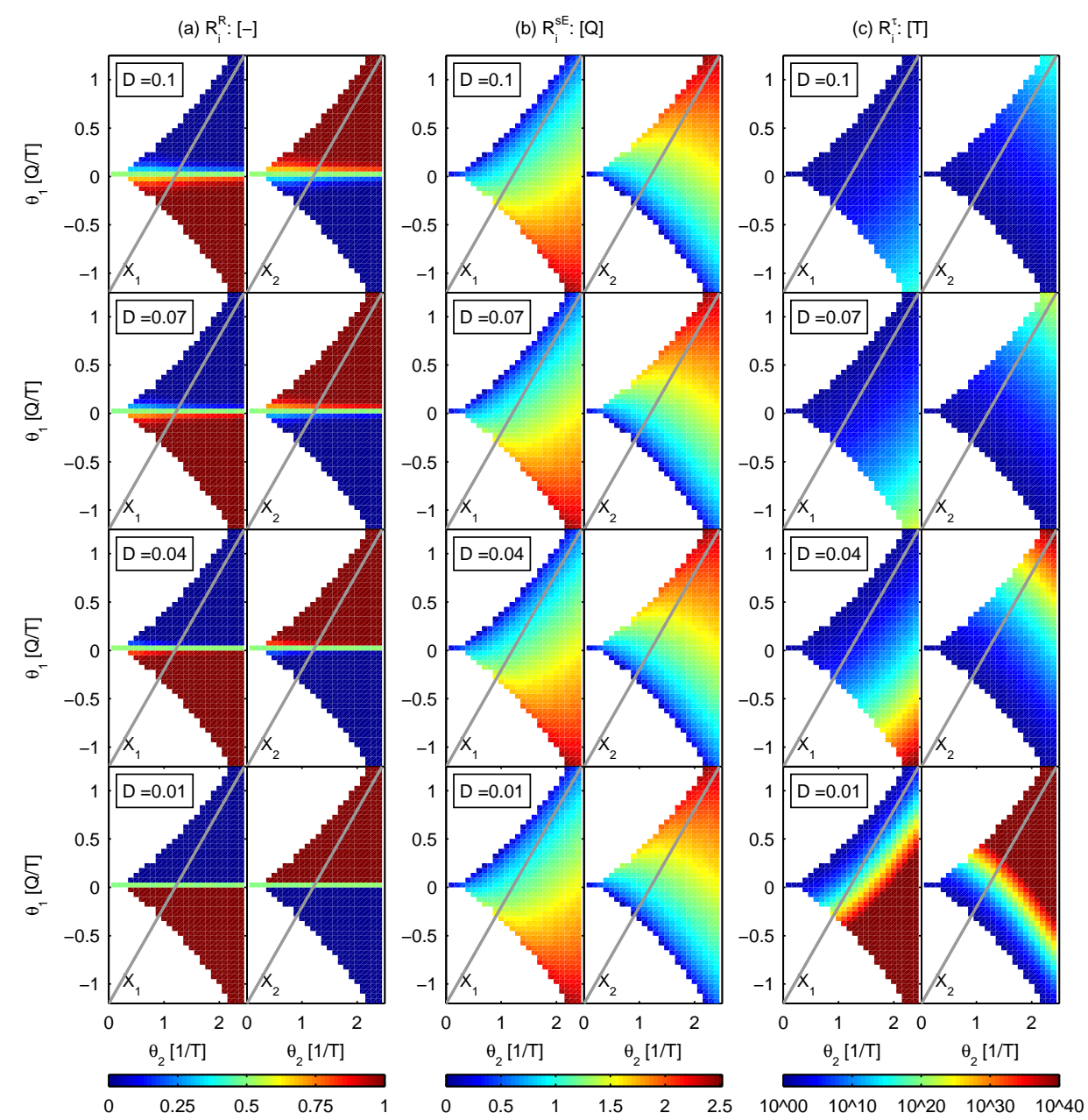

Figure 6: Effect of the variation of parameters $\theta_{1}$ and $\theta_{2}$ on resilience measures for Gaussian white noise variability in the system drivers at four different intensities of driver variability for the two dynamic regimes $X_{1}$ and $X_{2}$. Increasing the dirver variability $D$ results in a (a) diffusive effect in regime probability $\mathscr{R}_{i}^{R}$, an (b) invariant effect in stochastic ecological resilience $\mathscr{R}_{i}^{s E}$ and a (c) weakening effect in mean passage time $\mathscr{R}_{i}^{\tau}$. When $D=0.01$ at $\theta_{1}=0.05$ the values for $\mathscr{R}_{1}^{\tau}$ at $\theta_{2}=1.0$ and $\theta_{2}=2.0$ are $3.2496 \mathrm{e}+11$ and $5.9942 \mathrm{e}+43$ respectively (representing an increase of approximately 32 orders of magnitude), whereas when $D=0.1$ the values are $6.6265 \mathrm{e}+01$ and $5.1054 \mathrm{e}+04$ respectively (representing an increase of approximately 3 orders of magnitude). Note that for illustration purposes, the upper and lower bounds for the $\mathscr{R}_{i}^{\tau}$ plot in (c) have been cropped. The diagonal grey line indicates slopes of constant immigration or emigration rates $I$ [number/year] for the population dynamics example problem. 

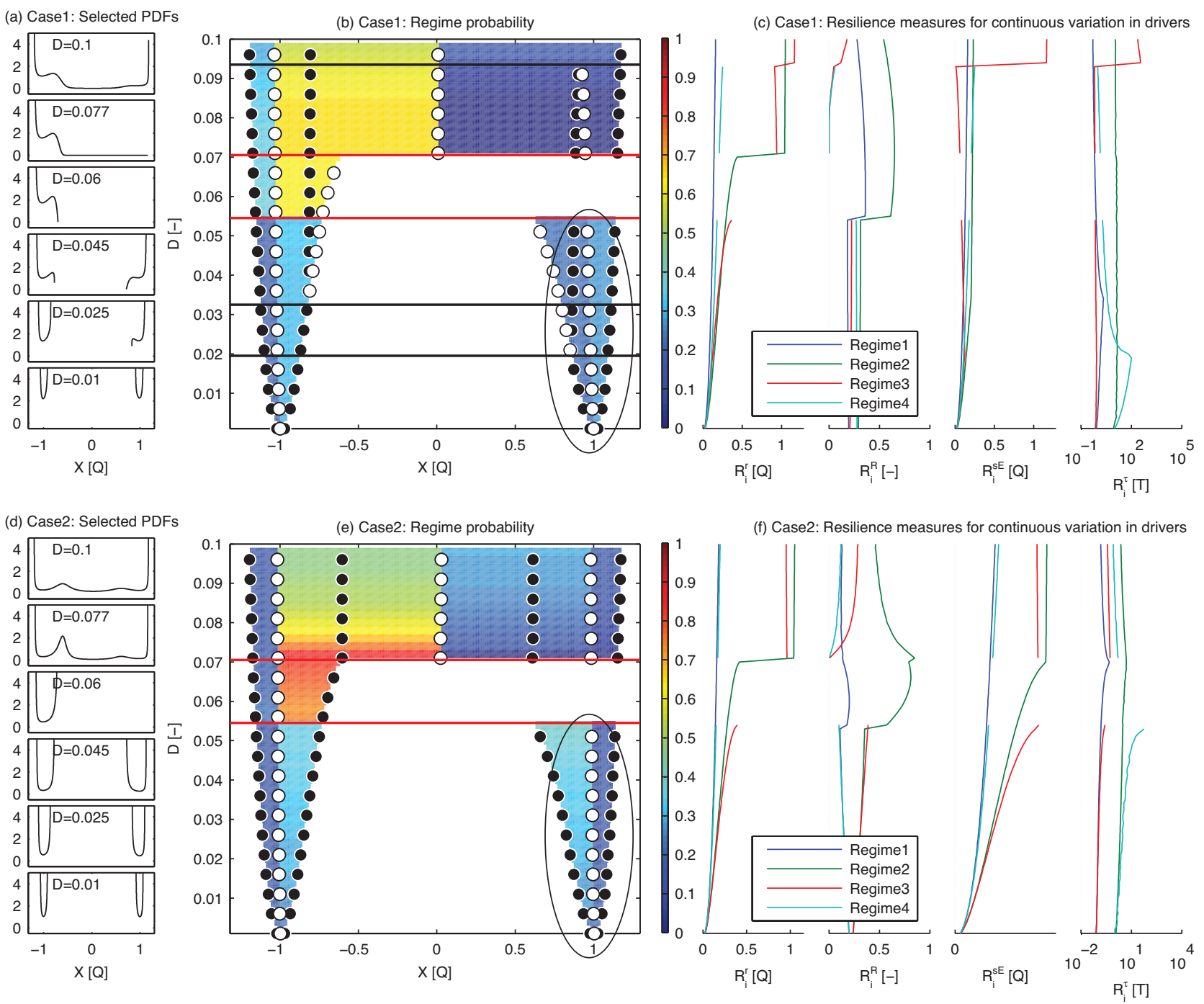

Figure 7: Noise induced bifurcation effect on resilience due to a Markovian dichotomous noise for a catastrophic shift system with parameters $\theta_{1}=0.01, \theta_{2}=1.0, \theta_{3}=-1.0, \Delta_{1}=-1.2, \Delta_{2}=1.0$. Case 1 (subplots a, b and c) represent $\gamma_{1}=1.2$ and $\gamma_{2}=1.0$ and case 2 (subplots $\mathrm{d}$, e and f) represent $\gamma_{1}=0.12$ and $\gamma_{2}=0.1$. (a and d) Sample PDFs for different magnitudes of driver variability $D$ illustrate the bifurcation in the PDFs. (b and e) Regime probabilities $\mathscr{R}_{i}^{R}$ (color coded) as a function of driver variability $D$ along with the locations of modes (black circles) and anti-modes (white circles) demonstrate noise induced bifurcations at four locations resulting in changes in the number of dynamic regimes. Bifurcations caused by $\Delta$ and $\gamma$ are indicated by horizontal red $(D=0.0545$ and $D=0.0705)$ and black $(D=0.01952, D=0.0325$ and $D=0.0935)$ lines respectively. The width of the individual color regions in (b and e) represents the size of the dynamic regimes $\mathscr{R}_{i}^{r}$. The barrier region lies below the bifurcation line at $D=0.0545$ and the region with trap or escape behavior is indicated by the ellipse. (c and f) Regime size $\mathscr{R}_{i}^{r}$, regime probability $\mathscr{R}_{i}^{R}$, stochastic ecological resilience $\mathscr{R}_{i}^{s E}$ and mean passage time $\mathscr{R}_{i}^{\tau}$ resilience measures as a function of driver variability $D$. 
(a) Time trajectory of $X$
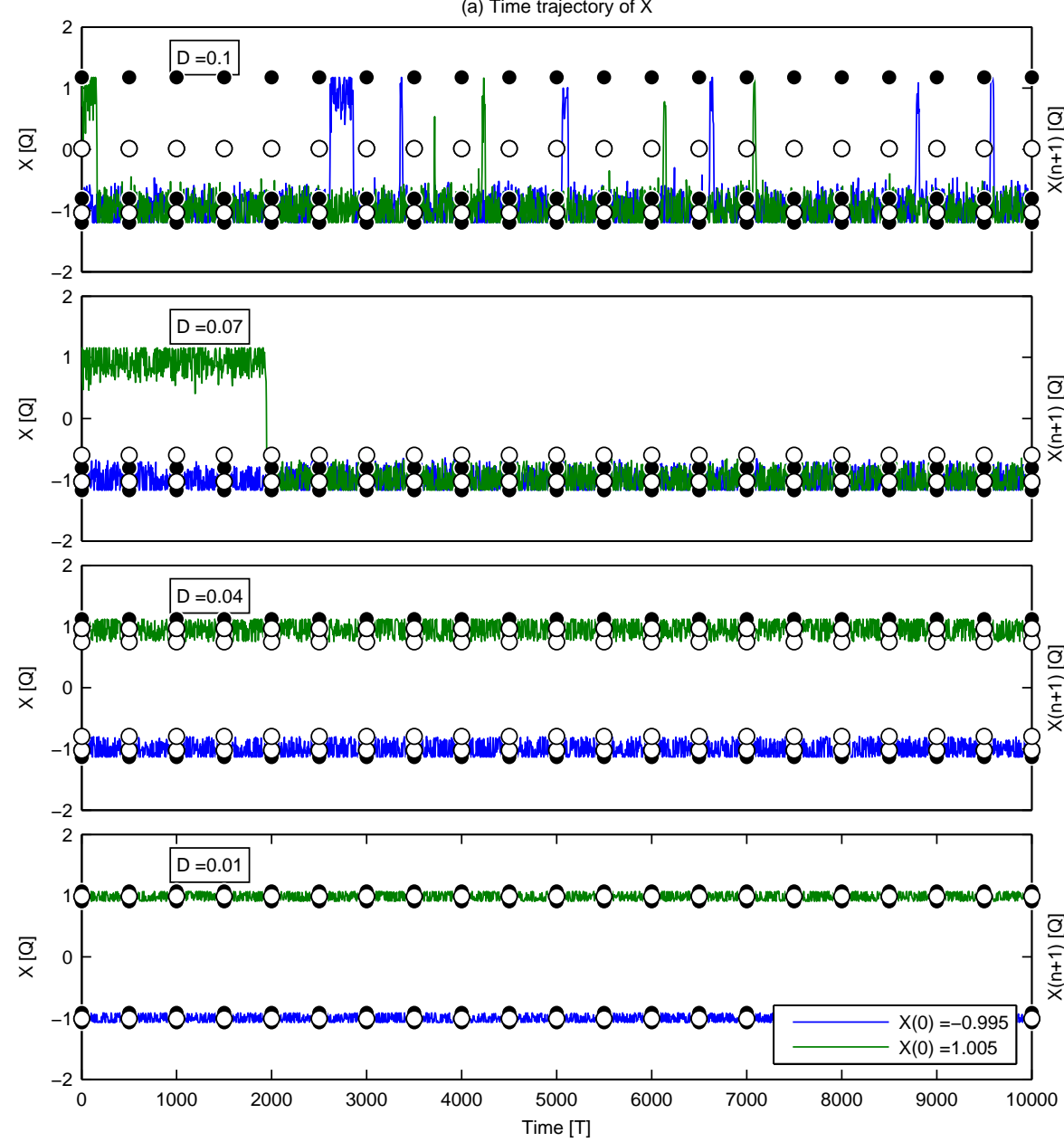
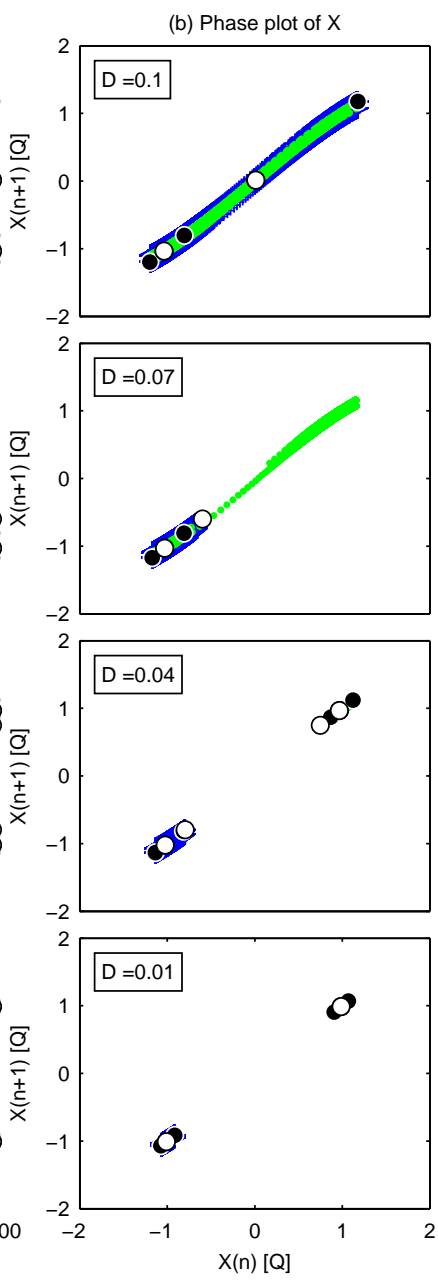

Figure 8: Effect of Markovian dichotomous noise variability in the system driver on the time trajectory of the system state $x$ at different intensities of driver variability on a catastrophic shift system with $\theta_{1}=0.01$, $\theta_{2}=1.0, \theta_{3}=-1.0, \Delta_{1}=-1.2, \Delta_{2}=1.0, \gamma_{1}=1.2$ and $\gamma_{2}=1.0$. Two different initial conditions at time $t=0$ are selected; the blue line corresponds to $x(0)=-0.995$ and the green line corresponds to $x(0)=1.005$. (a) Time trajectories for different magnitudes of driver variability $D$ illustrate the effect of trap regions $D=0.01$ and $D=0.04$, escape regions $D=0.07$ and connected regimes $D=0.1$. (b) Phase plots illustrating the range of values that the system state can take when subjected to Markovian dichotomous noise. The locations of the modes of the PDF are represented as black circles and the locations of the anti-modes are represented as white circles. 

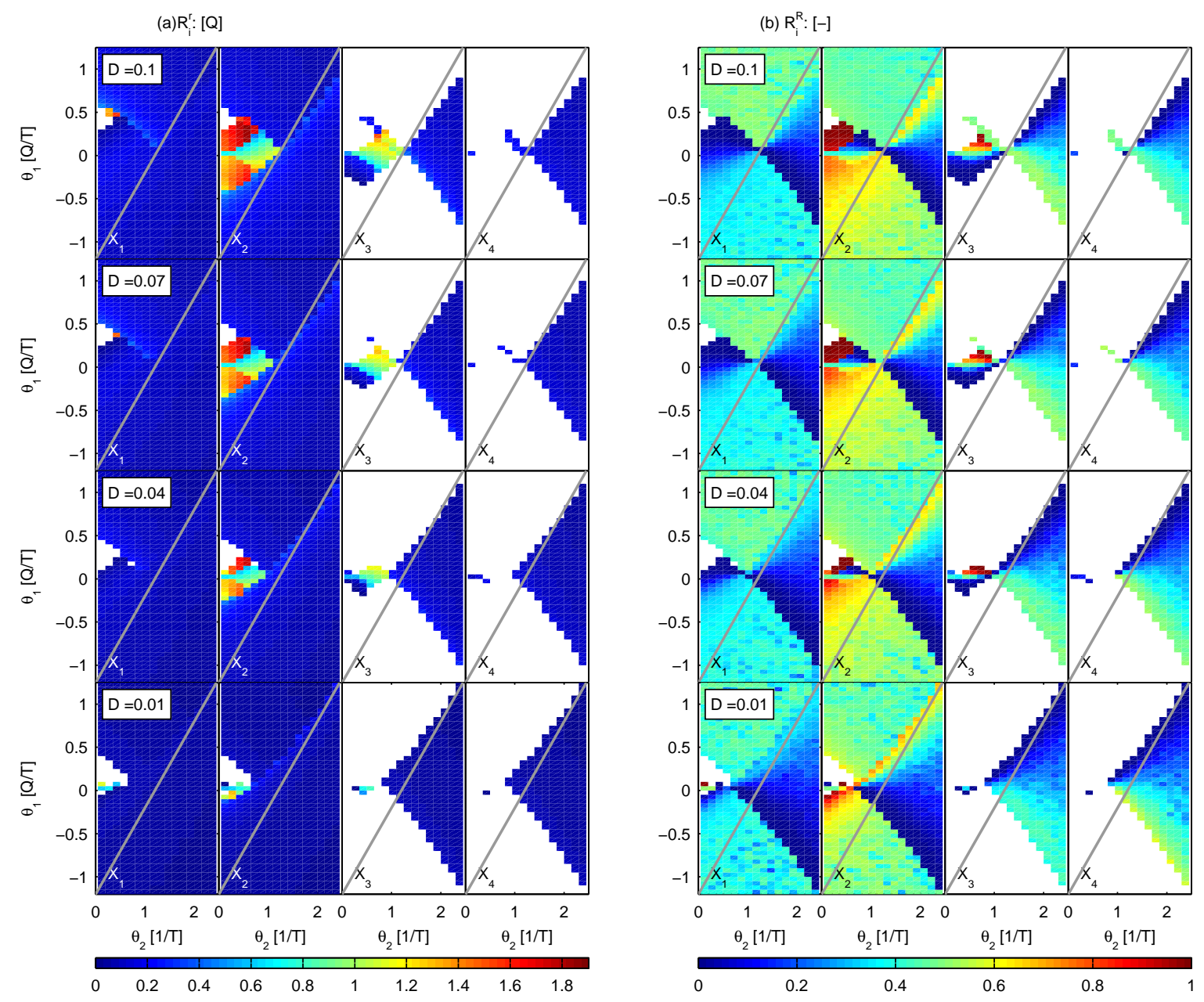

Figure 9: Effect of the variation of parameters $\theta_{1}$ and $\theta_{2}$ on resilience measures (a) Regime size $\mathscr{R}_{i}^{R}$ and (b) Regime probability $\mathscr{R}_{i}^{R}$ for Markovian dichotomous noise at four different intensities of driver variability for the four dynamic regimes $X_{1}, X_{2}, X_{3}$ and $X_{4}$. The other parameters used in this simulation were $\Delta_{1}=-1.2$, $\Delta_{2}=1.0, \gamma_{1}=1.2$ and $\gamma_{2}=1.0$. White spaces represent regions where the dynamic regimes do not exist. The effect of noise induced bifurcations can be observed with the changing locations of these white spaces across all four dynamic regimes when the driver variability $D$ is varied. The diagonal grey line indicates slopes of constant deforestation and reforestation rates $A\left[\mathrm{~m}^{2} /\right.$ year $]$ in Example B. 

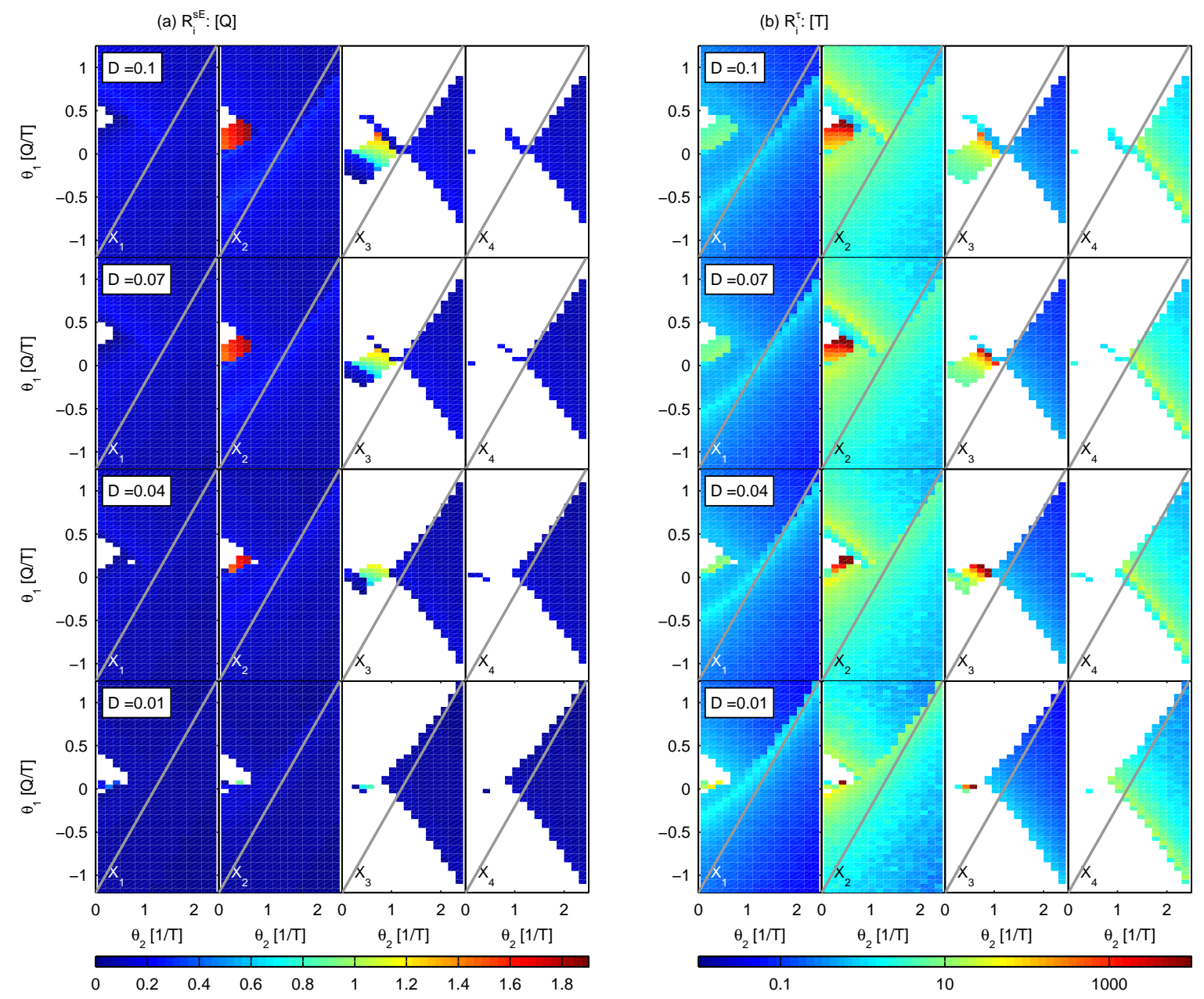

Figure 10: Same as figure 9 but for (a) Stochastic ecological resilience $\mathscr{R}_{i}^{s E}$ and (b) Mean passage time $\mathscr{R}_{i}^{\tau}$ measures. Note that for illustration purposes, the upper and lower bounds for the $\mathscr{R}_{i}^{\tau}$ plot in (b) have been cropped. 

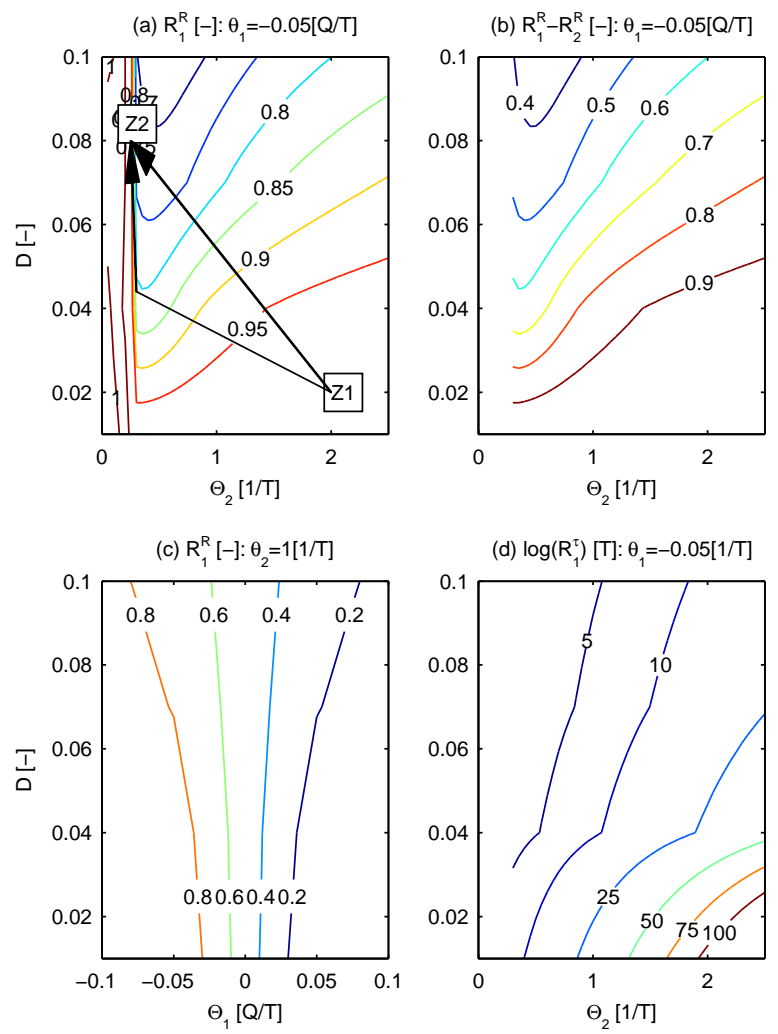

Figure 11: Iso-resilience curves of different resilience measures for a catastrophic shift system subject to Gaussian white noise as a function of driver variability $D$ and parameters $\theta_{1}$ or $\theta_{2}$. (a) regime probability of dynamic regime $1 \mathscr{R}_{1}^{R}[-]$, (b) regime dominance obtained as the difference in regime probabilities between dynamic regimes 1 and $2 \mathscr{R}_{1}^{R}-\mathscr{R}_{2}^{R}[-]$, (c) regime probability of dynamic regime $1 \mathscr{R}_{1}^{R}[-]$ and (d) natural logarithm of mean passage time $\log \mathscr{R}_{1}^{\tau}[T]$. Z1 and Z2 are the starting the ending points for state transition and the bent and the straight arrows represent transition paths with and without the constraint $\mathscr{R}_{1}^{R}>0.8$ respectively. 